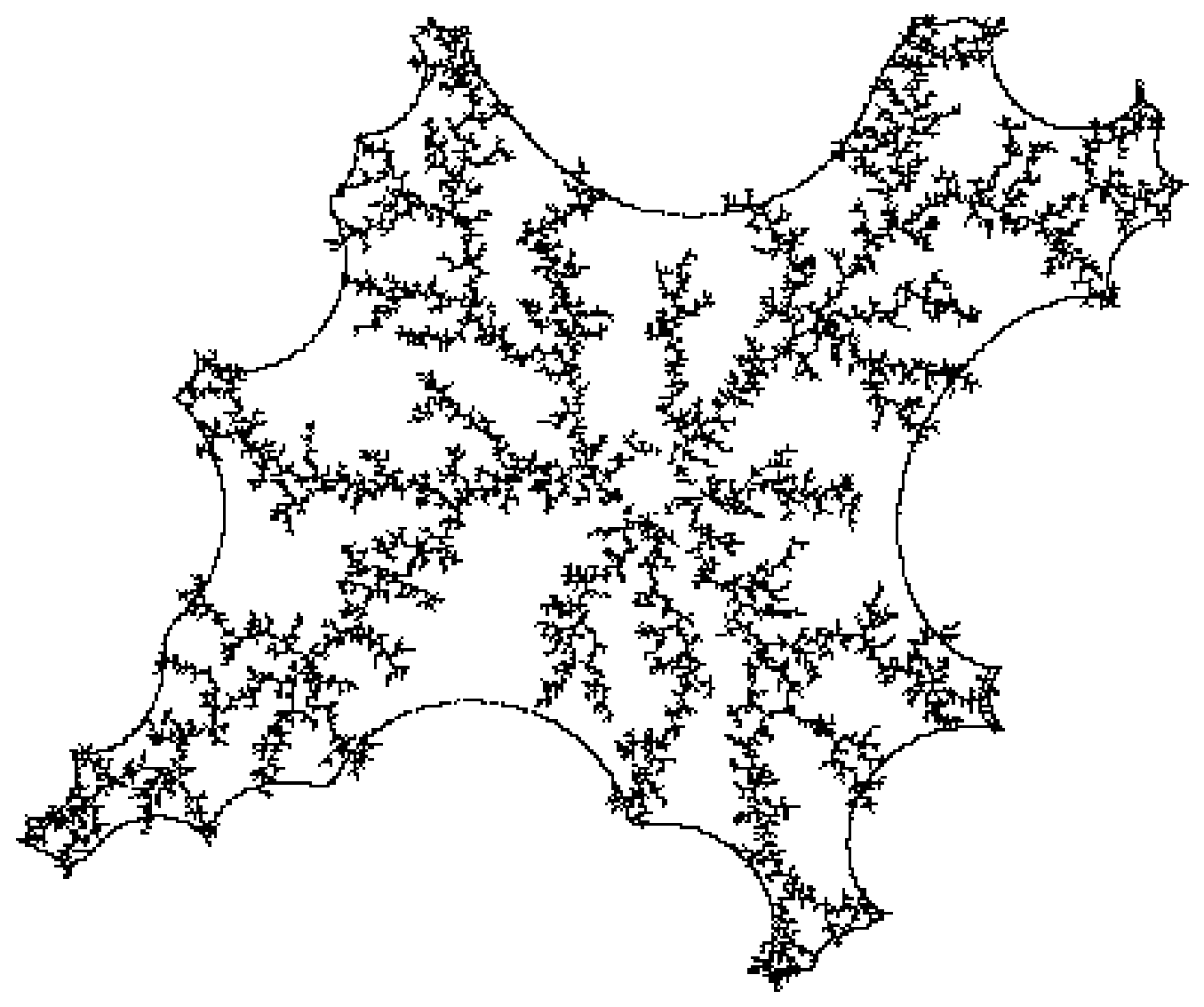




$$
0
$$


Fig. 3

a)

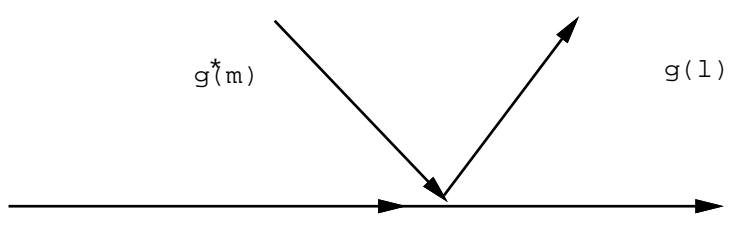

$g(j)$

b)

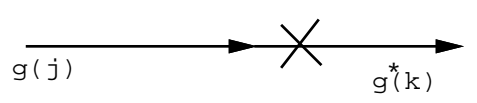


Fig. 4 Hastings

a)

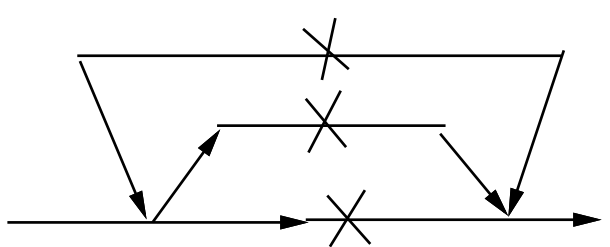

b)

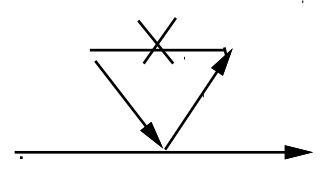

C)

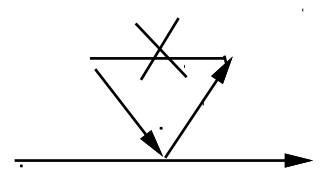


Fig. 5

Hastings

a)
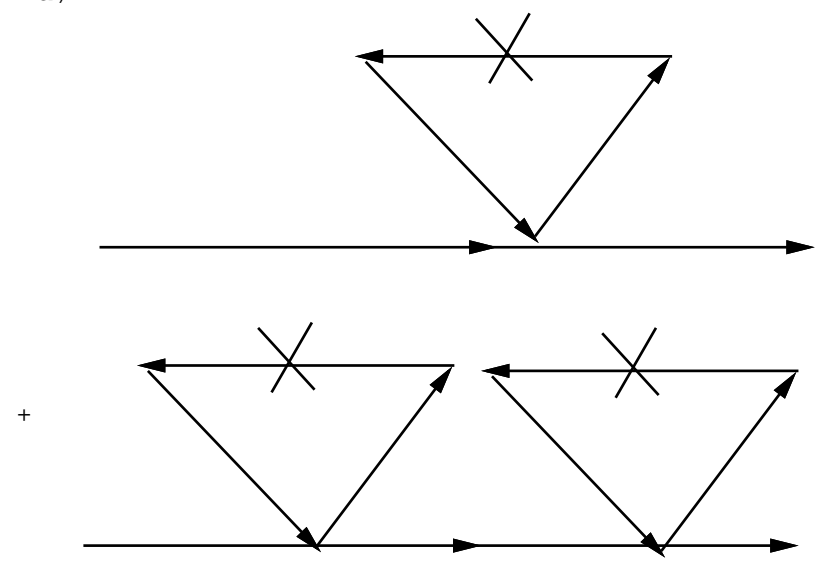

$+\ldots$ 
Fig. 6

Hastings

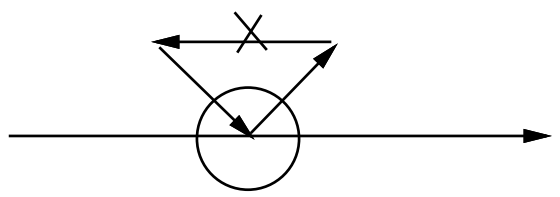


Fig. 7 Hastings

a)

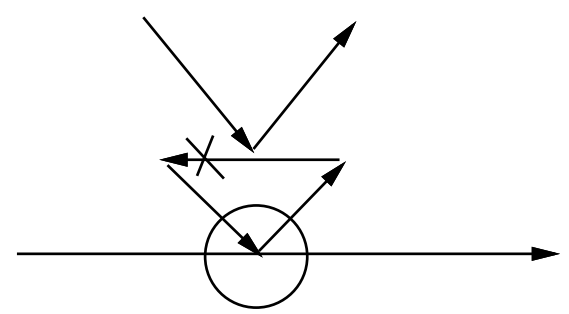

b)

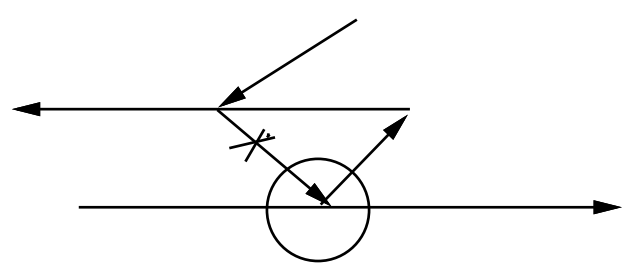


Fig. 8

Hastings

a)

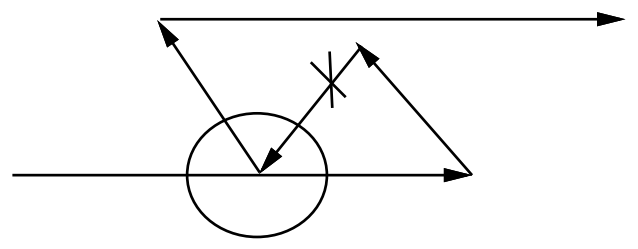

b)

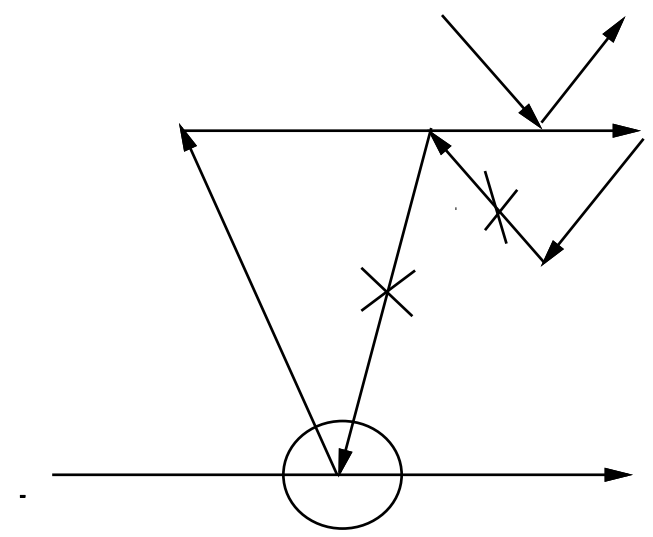

c)

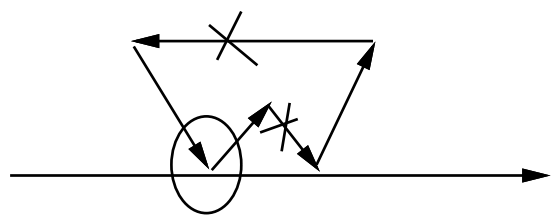


Fig. 8 Hastings

d)

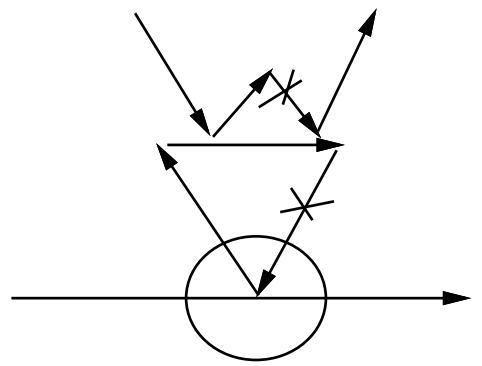

e)

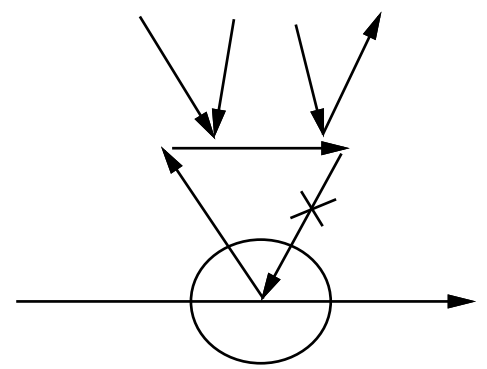

f)

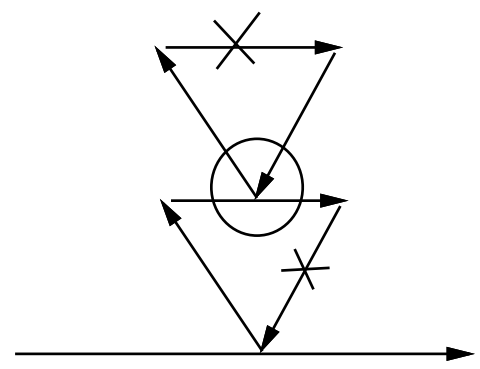


Fig. 9 Hastings

a)

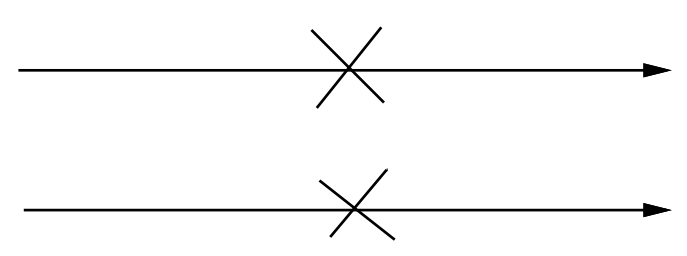

b)

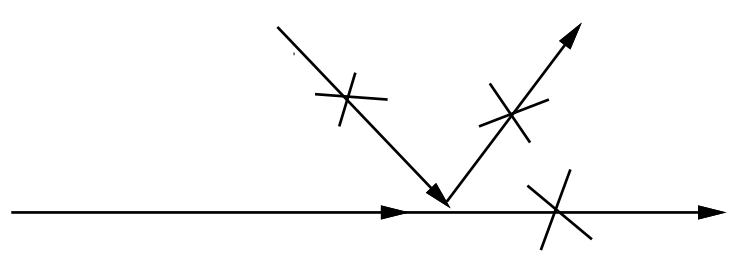


Fig. 10 Hastings

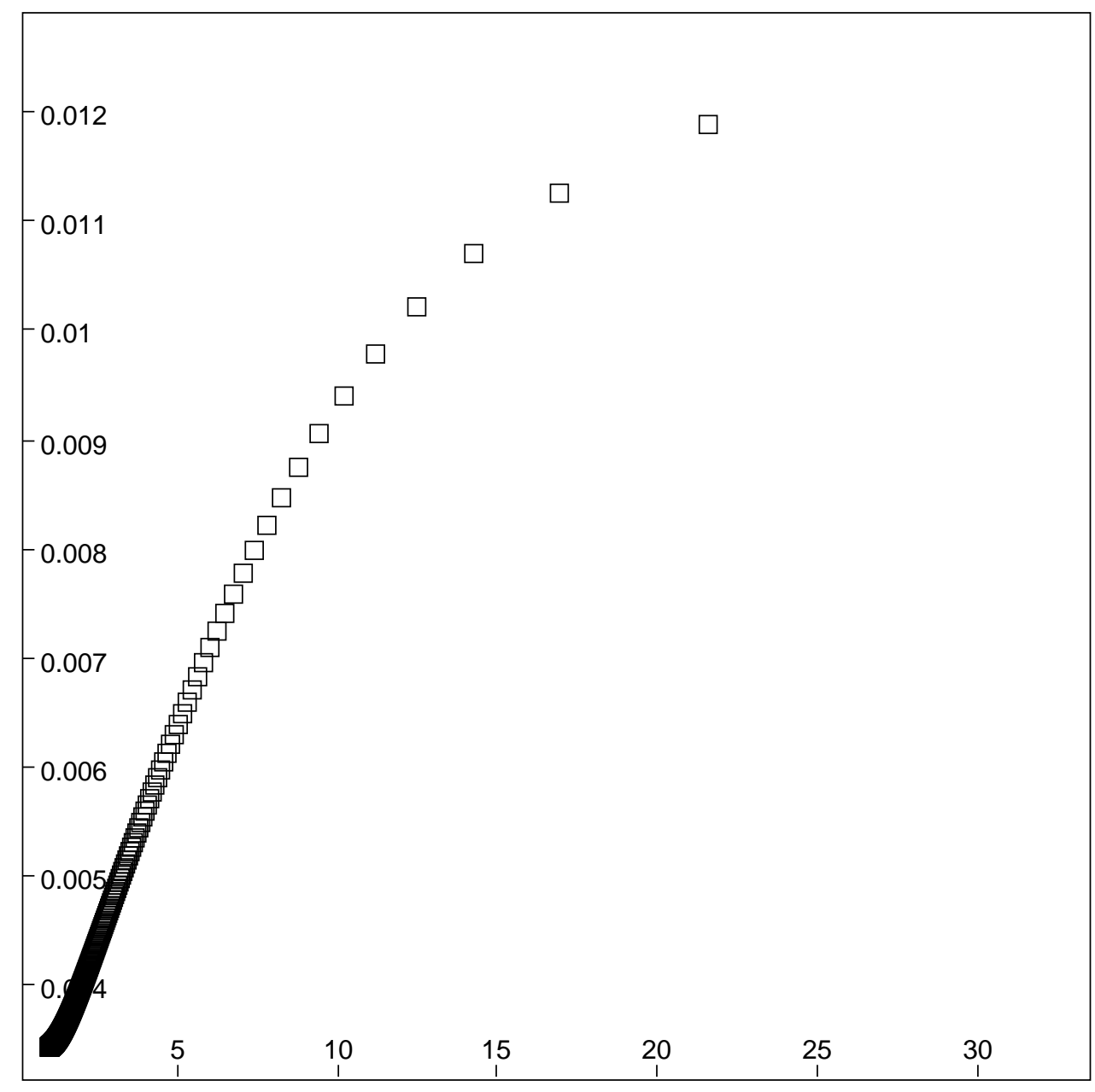


Fig. 11 Hastings

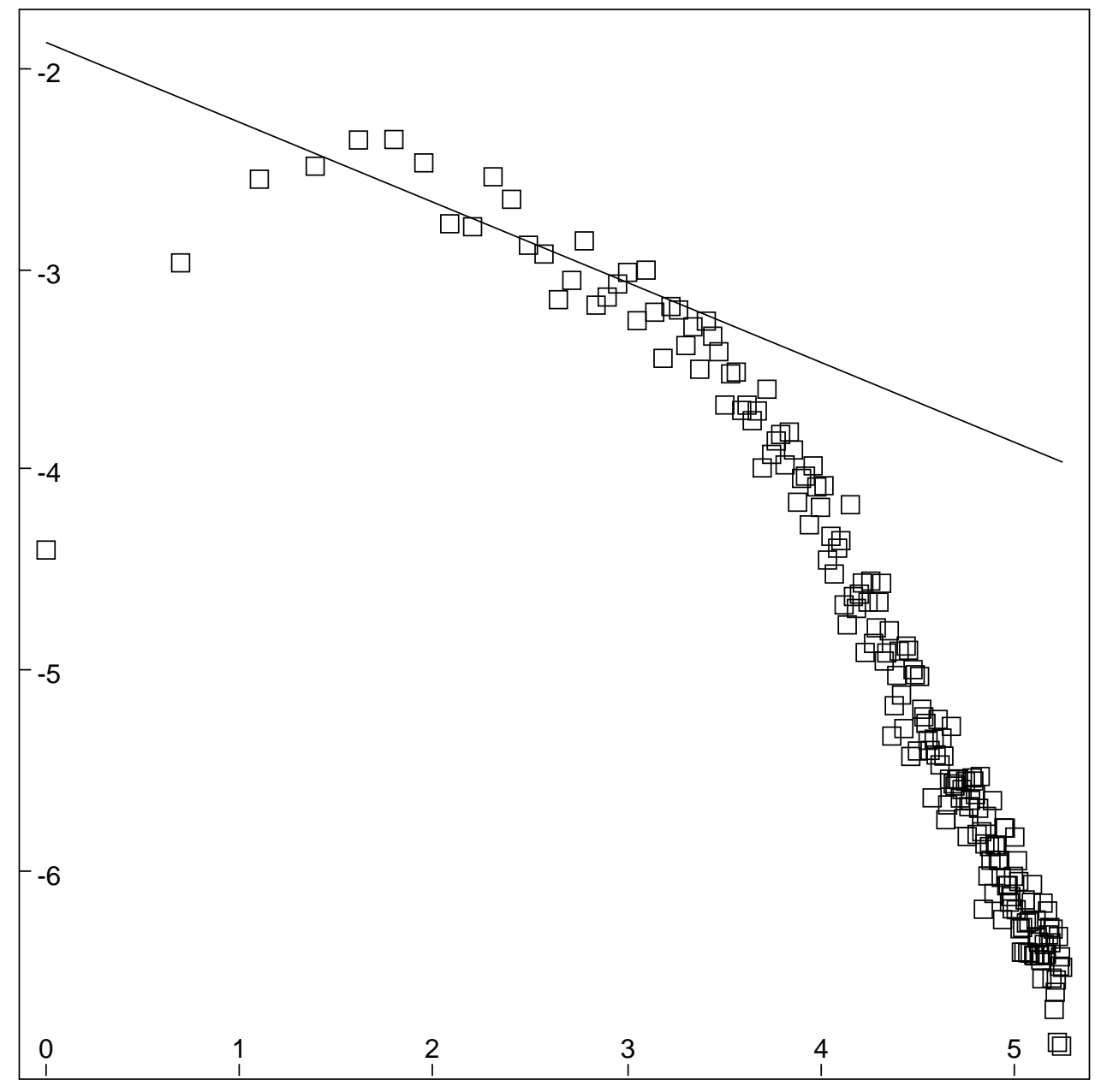




\section{Figure Captions}

1. Picture of a cluster grown using conformal growth model.

2. Illustration of effect of $f$ on the unit circle.

3. Diagrams illustrating interaction vertex and contraction of noise terms. The first diagram results from setting $G(j)=\widehat{W}\left[j, G(k), G(l), G^{*}(m)\right]$. The second diagram would occur in evaluating $\left\langle G^{f}(j) G^{f *}(k)\right\rangle=\delta(j-k) N(j)$.

4. a)A possible contribution to the average of $\left|G^{2}\right|$. b)A possible contribution to $s^{\text {eff }}$. c)A diagram which should not be considered if the propagators are already drawn in terms of $s^{e f f}$. 5. Perturbation expansion in the example theory.

6. Diagram contributing to the renormalization of $s^{e f f}$.

7. a) Diagram contributing to the renormalization of $t$. b)Another possible contribution to the renormalization of $t$. This contribution is ignored. Certain difficulties are encountered in drawing this diagram. If the $t$ interaction were drawn in standard form, many lines would need to cross each other.

8. Other possible contributions in the RG flow.

9. a)Simplest diagram for the correlation function needed to compute $\tau(5)$. The external lines are the various $G(j), G^{*}(k)$ in the correlation function. b) Tree diagram for the same correlation function.

10. Plot of scaling of $\lambda$ against cutoff, as described in text.

11. Plot of scaling of $\log$ of mean square of $G(j)$ against $\log$ of $j$, as described in text. 


\title{
Renormalization Theory of Stochastic Growth
}

\author{
Matthew B. Hastings
}

June 11, 2018

\begin{abstract}
An analytical renormalization group treatment is presented of a model which, for one value of parameters, is equivalent to diffusion limited aggregation. The fractal dimension of DLA is computed to be $2-1 / 2+1 / 5=1.7$. Higher multifractal exponents are also calculated and found in agreement with numerical results. It may be possible to use this technique to describe the dielectric breakdown model as well, which is given by different parameter values.
\end{abstract}

\section{Introduction and Notation}

\subsection{Introduction}

Diffusion limited aggregation (DLA) is a model for growth of a cluster [1], by the accretion of random walkers. These random walkers arrive from infinity and stick when they contact the cluster. After a walker sticks to the cluster, the next walker is released from infinity. This process gives rise to fractal patterns. Due to the mathematical equivalence of random walks and potential theory, this procedure is equivalent to solving Laplace's equation outside the boundary of the aggregate, setting the potential zero on the aggregate and constant at infinity, and picking a point on the surface of the aggregate to add the walker with a probability proportional to the local field strength; this field strength may be thought of as an "electric field".

There has been much numerical work on DLA in two dimensions, where the fractal dimension has been determined to be 1.71[2]. Mean-field calculations predict $D=5 / 3[3]$, which indicates that, in two-dimensions, something is lacking in the mean-field approach. In higher dimensions the mean-field theory appears much more accurate. One first principles 
renormalization theory, based on the branching nature of DLA processes, obtained the result that $D=1.661[4]$.

Another important analytic result was the derivation of the electrostatic scaling law, which appears to be obeyed numerically by the aggregates [5]. This law is used as an essential step in the calculation of this paper.

Recently, another formulation of DLA was proposed, based completely on conformal mappings [6]. A conformal function mapped the unit circle onto the boundary of the aggregate. In this formulation of the problem, the electrostatic scaling law followed almost automatically when considering the behavior of the first Fourier component of the mapping. We investigated numerically the problem of the importance of different Fourier components of the mapping. It appeared that by directly simulating the dynamics of only on the first few Fourier components, results could be obtained for short growth periods that were similar to those when the full function was used. This suggested that it might be possible to develop a renormalization theory based on integrating out higher Fourier components, using techniques similar to those used in field theory.

Also, by comparing a picture of the cluster generated by only keeping some small number of terms in the Fourier expansion of the mapping to a picture generated by the full mapping, it appeared that the finite number of terms gave a good description of the boundary of the object. It did not accurately describe the exact microscopic structure of the growth tips, and did not correctly describe the structure of portions of the object far from the growth region, that is, deep in the inside of the object. However, one would expect that the microscopic structure is not too important, and that the description of regions where there is little probability of growth is also not important.

Fig. 1 shows a picture of the cluster that results from the conformal mapping model. The envelope surrounding the cluster was generated from the first 40 terms of the Fourier series expansion of the mapping used to generate the full cluster.

For longer time periods, more terms in the Fourier series were needed, but this is only to be expected; if only one term were kept in the Fourier series the object would be a circle and would grow with a radius proportional to the square root of time. As more terms are kept, the object can grow faster than the square root of time by changing shape, but for any given number of terms, eventually the growth will be as the square root of time. Therefore, it is expected that 
in the RG that follows there will be some cutoff in the number of terms kept which increases with the size of the object.

The above discussion is intended to motivate the RG that follows. Most of the discussion is done in more detail in Ref. [6].

Hope, that such a scheme would work, was provided by numerical evidence that the conserved quantities (the moments) of the continuum growth law were very nearly conserved by the random growth process [7]. For the lowest Fourier coefficients, one would expect that the random growth would be close to the average growth determined by the continuum law, while the higher Fourier coefficients would fluctuate more wildly.

The paper is divided into several parts. First, the conformal mapping model for DLA is discussed and used to derive continuum equations for growth, essentially equivalent to the Shraiman-Bensimon equation for the Hele-Shaw problem [8]. These equations are heuristically modified to add the essential differences between DLA and its continuum limit: the presence of noise and the existence of a microscopic cutoff. This leads to a new model which is hoped to be in the same universality class as DLA. Even if it is not in the same universality class, it is similar enough to be of interest in itself.

Second, under an adiabatic assumption, the equation is modified to vastly simplify the time dependence, leaving almost a static problem. The adiabatic assumption makes possible the RG and perturbation theory calculations described latter in the paper. The adiabatic assumption is justified by numerics and self-consistently by the RG itself.

At this point, before doing the RG, it is still possible to make some comparison to numerics based on the continuum representation of DLA.

Third, a perturbation theory is developed for the continuum equation, with a well defined set of rules for calculating correlation functions. The perturbation theory requires some resummation of diagrams, where to calculate resummed propagators it is necessary to use a renormalization group approach. This RG forms the fourth part of the paper; the calculations for the RG have only been done to lowest order, producing an appropriate renormalized propagator and vertex. Fifth, the adiabatic assumption is removed, and the renormalized propagator is used to calculate various exponents in the theory. Sixth, the results are compared to numerical experiments, the self-consistency of the approach is discussed, and there is discussion of what may happen if the computation is performed to higher orders. 


\subsection{Notation}

A large number of functions will need to be defined in this paper. As much as possible, I will use the following notations. Capital letters are used for functions, such as $F, G$ to be defined latter, which describe the shape of a specific growing aggregate. Power series expansions of these functions will be denoted by subscripts, so $F(z)=F_{1} z^{1}+F_{0} z^{0}+F_{-1} z^{-1}+\ldots$ In the continuum limit of these power series expansions, to be appropriately defined latter, where sums are approximated by integrals, the Latin letters $j, k, l, m, n, o$ will be used as indices. One will see terms like $G(j)$.

Greek letters $\epsilon, \Lambda, \mu$ will be used for ultraviolet and infrared cutoffs in these continuum laws.

Greek letters $\alpha, \lambda_{0}$ will be used for various parameters in the models defined in this paper.

Lower case letters will be used for functions which define growth rules for the aggregate. These include the functions $f, s, t$ defined latter.

Latin letters $x, z$ represent points in the complex plane. The number $t$ represents time, either as a discrete number of steps or as a real number in a continuum limit. The numbers $\theta, \phi$ represent angles, while the function $\theta(j)$ is the step function.

Unless otherwise specified, subscripts attached to functions will be used to denote derivatives, thus $F_{x}$ is the derivative of $F$ with respect to $x$. As an exception to this, the expression $f_{\lambda, \theta}(z)$ will represent a function parametrized by $\lambda$ and $\theta$.

\section{Conformal Model and Continuum Growth Law}

A model for growth is defined. From this model, the Shraiman-Bensimon equation is derived for a function $F$ which maps the unit circle in the complex plane onto the boundary of the growing object. Defining

$$
G=\frac{1}{F_{z}}
$$

and making some approximations, we obtain a continuum growth rule, equation (32), which still includes effects of noise and finite cutoff. 


\subsection{Conformal Model}

The following model for DLA in terms of conformal mappings leads to results that are apparently numerically equivalent to DLA [6]. It should be noted that the RG in this paper relies upon a continuum approximation to this model; this continuum approximation could also have been obtained from the lattice version of DLA without reference to the conformal mapping model, but the conformal mapping model provides a better justification for the continuum equation.

In this method, we deal only with the analytic function $F$, which is defined as the analytic function which maps the unit circle in the complex plane onto the boundary of the growing cluster. We introduce two parameters, $\alpha$ and $\lambda_{0}$, where $\alpha=2$ corresponds to DLA and $\lambda_{0}$ is some constant determining the size of an individual random walker. To grow the object, first pick a random point $x=e^{i \theta}$ on the unit circle. Then, calculate $F_{z}(x)$, which is the derivative of $F$ at this point. We define

$$
\lambda=\lambda_{0}\left(F_{z}(x) F_{z}^{*}(x)\right)^{-\alpha / 2}
$$

The case of $\alpha=2$ will correspond to DLA and it is that case that will be considered from now on; other cases will be briefly discussed in the conclusion. Then in a given growth step, $F(z)$ is replaced by

$$
F\left(f_{\lambda, \theta}(z)\right)
$$

where $f$ is a function that produces a small bump at angle $\theta$, with linear dimension of the bump of the order of the square root of $\lambda . \lambda, \theta$ are parameters that define the function $f$. An explicit example of $f_{\lambda, 0}$ is given by:

$$
\left[\frac{1+\lambda}{2 z}(z+1)\left(z+1+\sqrt{z^{2}+1-2 z \frac{1-\lambda}{1+\lambda}}\right)-1\right]^{1 / 2} z^{1 / 2}
$$

For $\theta \neq 0$, we have $f_{\lambda, \theta}(z)=e^{i \theta} f_{\lambda, 0}\left(e^{-i \theta} z\right)$.

In the small $\lambda$ case, $f$ reduces to

$$
z+\lambda z\left(z+e^{i \theta}\right) /\left(z-e^{i \theta}\right)
$$

and by averaging over angle we may determine a continuum growth law. A picture of a cluster produced by this growth rule is shown in Fig. 1. A picture of the effect of the mapping $f$ on the unit circle is shown in Fig. 2. 


\subsection{Continuum Growth Law}

The continuum growth law for DLA is known to be equivalent to the Hele-Shaw dynamics, which obeys the law

$$
\operatorname{Im}\left(F_{t} F_{\phi}^{*}\right)=1
$$

where $F$ is a function which maps the unit circle in the complex plane onto the boundary of the aggregate and subscripts denote derivates with respect to time or to angle $\phi$ on the circle. This law may be rewritten as

$$
\begin{aligned}
& \operatorname{Re}\left(F_{t} / z F_{z}\right)=1 /\left(F_{z} F_{z}^{*}\right) \\
& \operatorname{Re}\left(F_{t} \frac{\left|F_{z}\right|}{z F_{z}}\right)=1 /\left|F_{z}\right|
\end{aligned}
$$

where here $z=e^{i \phi}$. Finally, this second growth law may be rewritten as

$$
F_{t}=F_{z} \int \frac{d \theta}{2 \pi}\left(F_{z}\left(e^{i \theta}\right) F_{z}^{*}\left(e^{i \theta}\right)\right)^{-1} z \frac{z+e^{i \theta}}{z-e^{i \theta}}
$$

This equation, the Shraiman-Bensimon equation, results from substituting the small $\lambda$ expansion of equation (5) into the equation (3) for the dynamics of $F$, where $F(f(z))$ is approximated by $F(z)+F_{z}(z)(f(z)-z)$.

In the Shraiman-Bensimon equation, one may divide both sides by $z F_{z}$ and then take the real part of both sides. This will recover equation (7) and show that the two equations are equivalent. Equation (7) implies that the normal velocity of the surface at a given point is proportional to the local electric field at that point.

Equation (5) may be rewritten as (taking $\theta=0$ for simplicity)

$$
z+\lambda z(z+1) /(z-1)=z+\lambda z\left(1+2 / z+2 / z^{2}+2 / z^{3} \ldots\right)
$$

Therefore, the effect of the integration over angle in the continuum growth law is to project out negative Fourier components in $\lambda$ considered as a function of angle. The factor of 2 difference between the zeroth component and all other components will be important later.

It will also be useful to define a continuum law for another function $G$ which is defined by equation (1). This function has several advantages. The equation for $\lambda$ then becomes

$$
\lambda=\lambda_{0} G(x) G^{*}(x)
$$

which has a simpler form than equation (2). This has a physical interpretation that $G$ determines the strength of the electric field at point $x$. Also, $G$ is the derivative of the inverse function 
of $F$ and the inverse function of $F$ has a more natural growth rule than $F$ does. That is, if $F^{-1}(F(z))=z$, then under a growth step with given $\lambda$ and $x$, the function $F^{-1}(z)$ changes into $f^{-1}\left(F^{-1}(z)\right)$. The continuum law for $G$, as obtained by using the definition of $G$ and the growth law for $F$, is

$$
\begin{aligned}
G_{t}= & G_{z} \int \frac{d \theta}{2 \pi} G\left(e^{i \theta}\right) G^{*}\left(e^{i \theta}\right) z\left(z+e^{i \theta}\right) /\left(z-e^{i \theta}\right) \\
& -G \int \frac{d \theta}{2 \pi} G\left(e^{i \theta}\right) G^{*}(e i \theta)\left[z\left(z+e^{i \theta}\right) /\left(z-e^{i \theta}\right]_{z}\right.
\end{aligned}
$$

It is also useful to consider the continuum growth laws for the power series of $F$ and $G(z)$. Writing

$$
\begin{gathered}
F(z)=F_{1} z^{1}+F_{0} z^{0}+F_{-1} z^{-1}+\ldots \\
G(z)=G_{0} z^{0}+G_{-1} z^{-1}+G_{-2} z^{-2}+\ldots
\end{gathered}
$$

then equation (11) is equivalent to

$$
\left(G_{-j}\right)_{t}=(j-2 k-1) \sum_{k, l, m} G_{-k} G_{-l} G_{-m}^{*} \delta(k+l-m-j) 2 \theta(j-k)
$$

where the discrete step function is defined by

$$
\theta(j-k)= \begin{cases}1 & \text { for } j>k \\ 1 / 2 & \text { for } j=k \\ 0 & \text { for } j<k\end{cases}
$$

An continuum equation may also be written for the power series expansion of $F$, but we will not need to use such an equation.

There are some problems with directly applying the continuum growth law above, in any of its forms, to the discrete random process that defines DLA. The continuum law leads to the appearance of cusps in the contour of the cluster after a finite time, and the continuum law is deterministic while the discrete law is random. However, the continuum law must have some applicability to the discrete cluster growth, because, for example, the conserved quantities of the continuum law are approximately conserved by the random process[]]. Thus, we will try, in the rest of this section, to correct the problems in the continuum law so that it may be of some use in describing the discrete, random growth process. 


\subsection{Ultraviolet Cutoff}

The above formulation of the problem suggests a method, outlined in this section, of inserting an ultraviolet cutoff into the growth law. This cutoff will be inserted by hand, and then the parameter of the cutoff will be adjusted to obtain the correct microscopic scale.

In the discrete conformal mapping model, $F$ never develops cusps because $f$ is always well behaved. The specific form of $f$ does not matter; all that matters is that the approximate expansion for $f$ given by $f(x)=z+\lambda z\left(1+2 / z+2 / z^{2}+\ldots\right)$ is only correct for small negative powers of $z$. The power series expansion of $f$ is cutoff at some point because $f$ is well behaved. This cutoff depends on $\lambda$, which itself depends on the angle $\theta$ at which growth is taking place. The approximation made in inserting the ultraviolet cutoff into the continuum law is that the cutoff in the power series expansion for $f$ depends only the average value of $\lambda$ over the circle at the time of a given growth step, and not on the exact value of $\lambda$ where the growth is occurring, where the dependence on the average value of $\lambda$ is chosen in such a way as to produce the correct microscopic scale in the DLA growth process.

Then a simplification follows. Suppose the regularized form of $f$ is chosen to be

$$
f(z)_{\lambda, \theta}=z+z \lambda \frac{(1+\epsilon) z+e^{i \theta}}{(1+\epsilon) z-e^{i \theta}}
$$

where $\epsilon$ is a fixed function of $\langle\lambda\rangle$, which is defined to be the average value of $\lambda$ over the entire circle.

It is worthwhile also to define

$$
\Lambda=1 / \epsilon
$$

Then $\Lambda$ represents the highest power of $z$ that will occur in the growth law.

Let us make a change of variable. We will replace $F(z)$ by the function $F\left((1+\epsilon)^{-1} z\right)$ and $G(z)$ by $G\left((1+\epsilon)^{-1} z\right)$. At the same time $f$ is replaced by $(1+\epsilon) f\left((1+\epsilon)^{-1} z\right)$. Then the continuum law (\$) for $F$ becomes

$$
F_{t}=F_{z} \int \frac{d \theta}{2 \pi}\left(F_{z}\left((1+\epsilon) e^{i \theta}\right) F_{z}^{*}\left((1+\epsilon) e^{i \theta}\right)\right)^{-1} z \frac{z+e^{i \theta}}{z-e^{i \theta}}
$$

where now the cutoff dependence has been moved to the derivatives of $F$. The continuum law (11) for $G$ becomes

$$
\begin{aligned}
G_{t}= & G_{z} \int \frac{d \theta}{2 \pi} G\left((1+\epsilon) e^{i \theta}\right) G^{*}\left((1+\epsilon) e^{i \theta}\right) z\left(z+e^{i \theta}\right) /\left(z-e^{i \theta}\right) \\
& -G \int \frac{d \theta}{2 \pi} G\left((1+\epsilon) e^{i \theta}\right) G^{*}\left((1+\epsilon) e^{i \theta}\right)\left[z\left(z+e^{i \theta}\right) /\left(z-e^{i \theta}\right]_{z}\right.
\end{aligned}
$$


The value of $\epsilon$ must now be calculated.

Before the averaging process, the dependence of the cutoff on $\lambda$ is easy to determine by, for example, expanding the function $f$ as defined by equation (4). After the averaging process, it is not necessarily the case that the average cutoff $\epsilon$ will be determined in the same way from the average value of $\lambda$. The averaging may introduce nontrivial behavior. Instead I will look for the dependence of $\epsilon$ on the cluster size; since the cluster size and the average of $\lambda$ are related, this is an equivalent procedure.

Expanding the cutoff in the continuum growth law to linear order in $\epsilon$ yields an additional term in the equation for $F_{t}$. This additional term changes equation (7) to

$$
\operatorname{Re}\left(F_{t} \frac{\left|F_{z}\right|}{z F_{z}}\right)=1 /\left|F_{z}\right|-2 \epsilon \operatorname{Re}\left(\frac{z F_{z z}}{F_{z}\left|F_{z}\right|}\right)
$$

The additional term may be written as

$$
2 \epsilon \operatorname{Im}\left(\frac{F_{\phi \phi}}{F_{\phi}\left|F_{\phi}\right|}\right)=2 \epsilon / R
$$

where $z=e^{i \phi}$ and $R$ is the local radius of curvature. This is a surface tension term.

The basic idea will be to adjust $\epsilon$ to produce the correct size for microscopic features; this size is the size of an individual walker in the lattice formulation of DLA.

A dimensional analysis argument may help understand the size of the cutoff. This dimensional analysis argument will relate the dependence of cutoff on macroscale to the dependence of cutoff on microscale.

The function $F(z)$ may be assigned the dimension of length, and $z$ may be made dimensionless. This means that we are interpreting $z$ as a parametrization of the cluster. Then both $\Lambda$ and $\epsilon$ are dimensionless. We know that $\Lambda$ must be a function of the size of an individual walker, but then since the dimensional argument implies that $\Lambda$ is dimensionless, $\Lambda$ must be proportional to some power of the ratio of the size of the object to the size of an individual walker, as this is the only way to form a dimensionless number. Let $r_{0}$ denote the length scale of an individual walker.

Let us see how to measure the linear size of the object. Recalling the expansion of $F$ in Fourier coefficients given by equation (12), by a theorem on univalent functions [9] the size of the object is at most 4 times $F_{1}$, the leading term of the power series. Asymptotically, $F_{1}$ will measure the size of the object. 
To fix the minimum radius of curvature at $r_{0}$, the size of an individual walker, the cutoff $\epsilon$ must be chosen so that $\epsilon / R$, the surface tension term, balances the electric field at the given radius. To determine the radius at which they balance, we need to make an assumption about the singularities of $G$. Let us assume $G$ has simple poles and therefore $F_{z}$ has simple zeroes. Suppose we look at points near a zero of $F_{z}$. Without loss of generality, take this zero to be at point $z_{0}$ where $z_{0}=1-\delta$ with $\delta$ some small positive number. Locally we find

$$
F_{z} \propto z-z_{0}
$$

The electric field at $z=1$ is proportional to $1 / \delta$. The radius of curvature at $z=1$ is proportional to $1 / \delta^{2}$. For the surface tension to balance the electric field we require

$$
1 / \delta=\epsilon / \delta^{2}
$$

This implies that $\delta=\epsilon$. Then, since $R=1 / \delta^{2}$, we need that $\epsilon=\sqrt{R}$. If $R$ is equal to $r_{0}$, we find

$$
\epsilon \propto r_{0}^{1 / 2}
$$

The dimensional argument then implies that $\epsilon \propto\left(r_{0} / F_{1}\right)^{1 / 2}$, where now the proportionality constant is dimensionless. This implies that

$$
\Lambda \propto\left(F_{1} / r_{0}\right)^{1 / 2}
$$

In the actual growth, $F_{1}$ is changing in time, but $r_{0}$ is constant. Thus the time dependence of $\Lambda$ is determined by $\Lambda \propto F_{1}^{1 / 2}$. As expected, the cutoff is increasing in time. If the power series expansion for $G$ is defined by equation (13), then

$$
G_{0}=1 / F_{1}
$$

so that it is also possible to measure the size of the object using the power series expansion of $G$.

As a further explanation of the dimensional analysis argument, it may be directly shown that, if the cluster is approximately circular, with a small bump on it, then the dependence of $\Lambda$ on the cluster size is correctly given by equation (25). The approximate circularity means that instead of simply stating that the electric field is proportional to $1 / \delta$ and that the radius of curvature is proportional to $1 / \delta^{2}$, we keep track of the proportionality constants in terms of 
$F_{1}$, and then directly show that $\epsilon \propto\left(r_{0} / F_{1}\right)^{1 / 2}$. The advantage of the dimensional argument is that it is possible to make this argument without any assumptions on the macroscale of the cluster; the dependence of $\Lambda$ on $r_{0}$ follows from microscopic considerations, and the dimensional analysis argument then yields the dependence on $F_{1}$.

One might worry that for the actual aggregate the poles will not necessarily be simple poles. As Halsey et. al. have shown [10], the surface is described by wedges with a non-zero opening angle, and the singularities exist on a fractal set. However, the continuum growth law of equation (11) only produces simple poles in $G$. It is only the dynamics that lead apparently to the creation of non-simple poles, via an accumulation of simple poles. Therefore, the cutoff will be imposed as if the poles were simple.

Because the object grows, $\Lambda$ increases with time. This is what leads to nontrivial dynamics and to a fractal dimension less than 2 . If instead of varying with time, the cutoff $\Lambda$ were held constant, then the aggregate would asymptotically grow at a $\Lambda$-dependent rate proportional to the square root of time, and would have a fractal dimension of 2 .

\subsection{Noise}

From now on, $G$ will be the function of interest and $F$ will be ignored. There are two reasons for this: the continuum law for $G$ is simpler, and noise may be more easily inserted into the law for $G$. The actual growth of $G$ is not deterministic; we may write the actual growth of $G$ symbolically as follows: actual growth of $G=$ continuum growth of $G+$ (actual growth of $G$ - continuum growth of $G$ ). The term in parenthesis represents noise. This noise term will be written as

$$
s G^{f}(z)
$$

where $s$ is a constant with dimensions of inverse time and $G^{f}$ is some function of $z$. We approximate that $G^{f}$ vanishes on average. If we expand $G^{f}$ in a series as $G_{-1}^{f} z^{-1}+G_{-2}^{f} z^{-2}+\ldots$, we will write the average of $G_{i}^{f} G_{j}^{f *}$ as

$$
\left\langle G_{i}^{f} G_{j}^{f *}\right\rangle=\delta_{i j} N(j)
$$

where $N$ is some unspecified function. We will also assume that any average of several $G^{f}$ can be written as a product of pairwise averages. These are the essential approximations in the noise. 
With noise included, we modify equation (19) to

$$
\begin{aligned}
G_{t}= & G_{z} \int \frac{d \theta}{2 \pi} G\left((1+\epsilon) e^{i \theta}\right) G^{*}\left((1+\epsilon) e^{i \theta}\right) z\left(z+e^{i \theta}\right) /\left(z-e^{i \theta}\right) \\
& -G \int \frac{d \theta}{2 \pi} G\left((1+\epsilon) e^{i \theta}\right) G^{*}\left((1+\epsilon) e^{i \theta}\right)\left[z\left(z+e^{i \theta}\right) /\left(z-e^{i \theta}\right]_{z}+s G^{f}(z)\right.
\end{aligned}
$$

The notation $G^{f}$ is used for the noise because, in the perturbation theory, the function $G^{f}$ will play a role similar to a free field in field theory.

The approximations are justified for two reasons. Since the noise is essentially generated by the dynamics, that is a small amount of noise will be amplified by the continuum growth, the dynamics should not be very sensitive to how the noise is inserted. This means we need not worry about the exact form of $N(j)$. Second, since the actual growth law for $F^{-1}$ is rather simple, involving a function acting on $F^{-1}$, it is most natural to insert the noise into $F^{-1}$, or into $G$, which is the derivative of $F^{-1}$. Inserting the noise into the growth law for $F$, which has a more complicated growth law, may have a different effect on the overall dynamics.

\subsection{Continuum Limit In Momentum Space}

In order to make the RG calculations easier, I will also take a continuum limit for the Fourier components of $G$ and $G^{f}$. This will result in replacing the discrete sums of equation (14) with integrals. This amounts to a change in the geometry of the growth; instead of parametrizing the boundary of the growing cluster by a point on the unit circle, we will parametrize it by a point on the real line in the complex plane.

In the neighborhood of a given point on the unit circle, such as $z=1$, the unit circle is locally approximated by a straight line. As we look at shorter and shorter length scales, this

approximation becomes more and more accurate. The equation $z=e^{i \theta}$ is approximated by $z=1+i \theta$. Thus, on short scales, in the neighborhood of $z=1$ we can approximate

$$
\begin{gathered}
F(z)=F_{1} z^{1}+F_{0} z^{0}+F_{-1} z^{-1}+\ldots \approx F_{1}(1+i \theta)+F_{0}+\int d j F(j) e^{-i j \theta} \\
G(z)=G_{0} z^{0}+G_{-1} z^{-1}+G_{-2} z^{-2}+\ldots \approx G_{0}+\int d j G(j) e^{-i j \theta}
\end{gathered}
$$

where $F(j)$ and $G(j)$ should be considered as being defined by the above equations. They are defined so that $j$ is always a positive quantity. This approximate form for $G(z)$ will break down for $\theta$ of the order of 1 radian. This implies that the Fourier expansions will break down for low values of $j$. This has the effect of an infrared cutoff; the cutoff will be at $j$ of order $\mu$, which 
is a number of order 1 . The cutoff $\mu$ is constant in time, unlike $\Lambda$, but under the RG we will find it convenient to rescale $\mu$. After introducing the equation of motion appropriate to this approximate expression for $G$ I will then explain the effect of nonzero $\mu$ on this equation.

The symbol $j$ in equations (30), (31) will be referred to as a momentum since it will play a role in the perturbation theory of the next section equivalent to that of a momentum in a perturbation theory for a field theory.

We take equation (14) and transcribe it to this continuum approximation. Noise is added as in equation (29). The result is:

$$
\begin{aligned}
G_{t}(j)= & (1 / \mu) \int d k t(j, k) G(k) \iint d l d m G(l) G^{*}(m) \exp (-(l+m) / \Lambda) \\
& \times \delta(k+l-m-j) 2 \theta(j-k)+s G^{f}(j)
\end{aligned}
$$

where $t(j, k)$ is some general function (initially it is proportional to $(j-2 k-1)$ as in equation 14), and where the factor of $1 / \mu$ is inserted to produce the correct dimensions for $t$ in the RG, as will be clear later. The insertion of the factor of $1 / \mu$ simply amounts to a redefinition of $t(j, k)$. The function $t$ will flow under the renormalization group. The exponential term is the appropriate version of the ultraviolet cutoff in the continuum limit. We define the continuous step function $\theta$ with by the same equation (15).

The effect of the cutoff $\mu$ is twofold: the $\delta$-function in equation (32) has a nonzero width of order $\mu$, and hence a finite height. The quantity $\delta(0)$ is of order $1 / \mu$. Also in the definition of the noise, equation (28) is replaced by:

$$
\left\langle G^{f}(i) G^{f *}(j)\right\rangle=\delta(i-j) N(j)
$$

where again the $\delta$-function has a nonzero width.

By rescaling momentum, the cutoffs $\mu$ and $\Lambda$ may be changed, but the ratio of the two cutoffs will remain constant. The purpose of the RG will be to integrate the upper cutoff from $\Lambda$ to $\Lambda-\delta \Lambda$. Then, for the sake of convenience, the upper cutoff will be rescaled back to $\Lambda$. The assumption is made that when $\Lambda$ is much greater than $\mu$ this renormalization does not change the essential physics of the system.

Physically, equation (32) describes the problem of DLA growth in the upper half of the complex plane, where the boundary of the growth is parametrized by a point $\theta$ on the real axis. The cutoff $\mu$ has the physical interpretation that growth only occurs in a finite width on this axis. 
For use later, let us define a functional $\widehat{W}$ such that

$$
\begin{aligned}
\widehat{W}\left[j, G(k), G(l), G^{*}(m)\right]= & (1 / \mu) \int d k t(j, k) G(k) \iint d l d m G(l) G^{*}(m) \exp (-(l+m) / \Lambda) \\
& \times \delta(k+l-m-j)
\end{aligned}
$$

Thus, the right hand side of equation (32) is $\widehat{W}\left[j, G(k), G(l), G^{*}(m)\right]+s G^{f}(j)$. The functional $\widehat{W}$ is linear in each of its last three arguments.

\section{Adiabatic Assumption and Numerical Predictions}

\subsection{Adiabatic Assumption}

An important approximation is made, which changes the problem to one of describing an aggregate which is statistically unchanging in time. In the end, we will describe aggregates whose average size and roughness remain constant.

The cutoff $\Lambda$ is slowly changing in time. As the object grows, $\Lambda$ changes more and more slowly. The exact structure of the cluster at a given time depends upon its growth at all previous times, but since the object spends a long time growing with an approximately fixed cutoff, it is expected that the structure of the object at a given time $t$ with resulting cutoff $\Lambda$ is determined only by its growth during previous times $t^{\prime}$ with resulting cutoffs $\Lambda^{\prime}$ such that $\Lambda^{\prime}$ is very close to $\Lambda$. Times $t^{\prime}$ such that $\Lambda^{\prime}$ is very different from $\Lambda$ will be so far in the past that we do not expect them to alter the structure of the cluster.

Furthermore, if the cutoff is fixed, the equations of motion are homogeneous, in the sense that up to a rescaling of time and noise, two clusters, which differ only by a change of scale, will have exactly the same growth for the same random noise. More precisely, if $G(t)$ is a solution of the equation (29), with fixed cutoff $\Lambda$ and given noise $G^{f}$, then, for any number $b$, the function $b G\left(b^{2} t\right)$ is a solution of equation (29) with the same cutoff $\Lambda$ and with noise $b G^{f}$ and $s$ replaced by $b^{2} s$.

For a large object it is then reasonable to make the adiabatic assumption that, despite the changing cutoff, up to a rescaling of the cluster, the statistical properties of the function $G$ at some given time with some given cutoff $\Lambda$ are well described by evolving an arbitrary initial $G$ for sufficiently long time using the equations of motion with the cutoff held fixed at that value $\Lambda$. 
First, we will analyze the dynamics of $G$ in the fixed cutoff problem, and then we will use the adiabatic assumption to relate it to the changing cutoff problem.

Let us take equation (32) and, holding the cutoff fixed, make a dynamical rescaling of the function $G$ as it evolves under this equation. After every time step of length $d t$ we will rescale $G$ to $G(1-s d t)$. Then, the rescaled $G$ satisfies the equation of motion

$$
\begin{aligned}
s G(j)+G_{t}(j)= & (1 / \mu) \int d k t(j, k) G(k) \iint d l d m G(l) G^{*}(m) \exp (-(l+m) / \Lambda) \\
& \times \delta(k+l-m-j) 2 \theta(j-k)+s G^{f}(j)
\end{aligned}
$$

On average, the rescaled $G$ has constant size.

The amount by which the cluster is rescaled per unit time, $s$, is the same $s$ referred to in the section on Noise. It is simply a matter of notational convenience to chose these two numbers $s$ to be the same. Any other choice of $s$ in the section on noise simply amounts to a redefinition of $G^{f}$.

The function $G$ before rescaling is growing in time. To determine how rapidly an unrescaled cluster of given size and given, fixed cutoff grows, we may follow this procedure: evolve a rescaled cluster using equation (35) with an $s$ chosen such that the rescaled cluster is of the desired size. Then, from the value of $s$ needed to maintain the desired size, determine the growth rate of the unrescaled cluster. For the unrescaled cluster, the average of $\frac{d \log \left(G_{0}(t)\right)}{d t}=s$.

Under the adiabatic assumption, we can now use the growth rate for the fixed cutoff problem, this growth rate being a function of the size of the cluster and the cutoff, and use it to determine the growth rate for a cluster with a changing cutoff. To determine the growth rate of a cluster of given size in the changing cutoff problem one can determine the cutoff from the size of the cluster and then calculate the growth rate of a cluster of the same size in the fixed cutoff problem, using the rescaling trick to determine how quickly that cluster grows.

Under RG scaling, in fact, we will find that the equation of motion changes in such a way that $s$ changes; in fact, $s$ may acquire momentum dependence. Before RG scaling $s$ will be negative, since $F$ is increasing, causing $G$ to decrease. After RG flow, an appropriate combination of $t, s$, and $G^{f}$ goes to a universal value. The adiabatic assumption will mean that we assume that at every instant in the original DLA problem, the function $G$ is described by a function in the long time limit of the problem with a fixed cutoff and a rescaling term $s$, where $s$ is picked to obtain the correct overall scale for $G$.

On average, $G_{t}$ in equation (35) vanishes. What is left of $G_{t}$ after the rescaling process 
is just fluctuations about the average growth. There may in fact be solutions such that $G_{t}$ vanishes identically, but this is unimportant. In the $\mathrm{RG}$, even with $G_{t}$ non-zero, the $s$ term and interaction term $\left(G^{3}\right.$ term) will determine the nature of the aggregate. As will be shown, under RG flow, the $G_{t}$ term flows under RG so that, the fluctuations in $G_{t}(j)$ decrease as $j$ decreases. The lower momentum terms then, in the unrescaled problem, will have their growth more accurately given just by the $s G$ term. Fluctuations about this overall growth will be less.

\subsection{Numerical Predictions}

It will be worthwhile to mention at this point that already some definite numerical predictions can be extracted from the above work. Since the RG that follows relies upon the continuum equations, it is good to independently check the validity of these equations for describing DLA.

If any RG is to hold, the coefficients of $G$ must obey some scaling law. In the last section of this paper, such a scaling law will be shown numerically. The coefficients decay with a power law. It will be the purpose of the RG to calculate this power law.

Since the absolute value of $G$ is equal to the local electric field, there is a close connection between $G$ and the multifractal exponents of references [12, 13, 14]. These exponents are defined by equations $(69),(73)$. The $(2 n+1)$-th power of the electric field, integrated over the object, is given by $\int d \theta\left(G(\theta) G^{*}(\theta)\right)^{n}$. This integral over $\theta$ can be converted to an integral over components of $G$ in momentum space. Cutting those off at momenta $\Lambda$ is equivalent to cutting the real space integration off at a length scale of order $\Lambda^{-2}$. Since the multifractal exponents are defined in terms of the scaling of powers of the electric field against length, we obtain an equivalent definition of multifractal exponents in terms of scaling of powers of $G$ against cutoff. This discussion of exponents will be done in more detail later, after the RG permits us to calculate these exponents analytically.

As a check of the adiabatic assumption, a numerical simulation was performed using the original discrete conformal mapping model defined in section (1.2). In this simulation, after every growth step, the object was shrunk by some constant factor. Visually we could not see any difference, in the growing region on the surface, between the cluster shrunk after every growth step and another cluster which was not shrunk. A calculation of fractal dimension also failed to show any significant differences. 


\section{Perturbation Theory}

A perturbation theory is developed for the equation of motion (35). This permits in principle the calculation of any correlation function of the theory in terms of noise averages. In practice, a resummation of the series is employed which expresses multi-point correlation functions in terms of two-point correlation functions.

\subsection{Perturbation Rules}

Using the adiabatic assumption, the equation of motion (35) looks very much like the variation of an action. Although there no such action can be found, a perturbation theory will be developed, based on this analogy, to permit the use of techniques from field theory.

This technique is very similar to that used, for example, in solving the Navier-Stokes equation [15]. Such a perturbation theory has been known for many years. Before proceeding with the details, let me summarize the essential attributes: a perturbation theory is developed by expanding $G$ in powers of the noise, $G^{f}$, and expanding correlation functions of $G$ in terms of twopoint correlation functions of the noise. Since the noise is amplified by the dynamics of equation (35), this expansion is not expected to converge. However, after resummation of the series, it becomes possible to replace this by an expansion not in the two-point correlation function of the noise, but in the two point correlation function of $G$ itself. Further, a resummation of series leads to a resummed propagator (defined below). Unlike the Navier-Stokes perturbation theory for turbulence 15], it will not not be necessary to define a resummed interaction vertex. The above procedure leads to a well defined perturbation series in "skeleton" diagrams. One point that will be necessary in the following treatment that is not necessary in the case of turbulence is that $G$ is a complex field, and thus the propagators will be directed lines; the notation that follows will therefore differ from that seen in Navier-Stokes problems.

The perturbation theory is constructed as follows: the equation of motion (35) permits us to solve for $G(j)$ in terms of a cubic in $G(j)$. Using the definition of $\widehat{W}$ in equation (34) we write

$$
\left.G(j)=\left(s(j)+\frac{\partial}{\partial t}\right)^{-1}\left(\widehat{W}\left[j, G(k), G(l), G^{*}(m)\right]+s(j) G^{f}(j)\right)\right)
$$

where $s(j)$ is used instead of $s$ because $s$ may, under RG flow, acquire $j$ dependence.

The operator $\left(s(j)+\frac{\partial}{\partial t}\right)^{-1}$ may be expanded as a formal power series in $\frac{\partial}{\partial t}$. In Navier- 
Stokes perturbation theory, this operator is referred to as the propagator, in analogy with a similar object in field theory.

We may then iteratively solve equation (36) as follows

$$
\begin{aligned}
G(j)= & \frac{1}{s(j)} \widehat{W}\left[j, G(k), G(l), G^{*}(m)\right]+G^{f}(j)+\mathrm{O}\left(\frac{\partial}{\partial t}\right) \\
= & \frac{1}{s(j)} \widehat{W}\left[j, G^{f}(k), G^{f}(l), G^{f *}(m)\right]+G^{f}(j) \\
& +\frac{1}{s(j)} \widehat{W}\left[j, \frac{1}{s(k)} \widehat{W}\left[k, G(n), G(o), G^{*}(p)\right], G^{f}(l), G^{f *}(m)\right] \\
& +\ldots \\
& +\mathrm{O}\left(\frac{\partial}{\partial t}\right) \\
= & \ldots
\end{aligned}
$$

The iterative solution of equation (36), by solving for the values of $G(k), G(l)$, and $G^{*}(m)$ on the right hand side, is an expansion in powers of the interaction, $t$. This is simultaneously an expansion in powers of the noise, $G^{f}$. The zeroth order and first order terms in $t$, and one of the second order terms, have been written in equation (37). At any point in the process, one can stop the perturbation expansion by setting $G(j)=G^{f}(j)$ plus higher orders in $\frac{\partial}{\partial t}$. Thus, in the perturbation expansion for $G$ one sums at every stage over two possible expressions for $G$ :

$$
\begin{aligned}
G(j)= & (1 / s(j))(1 / \mu) \int d k t(j, k) G(k) \iint d l d m G(l) G^{*}(m) \exp (-(l+m) / \Lambda) \\
& \times \delta(k+l-m-j) 2 \theta(j-k)+\mathrm{O}\left(\frac{\partial}{\partial t}\right)
\end{aligned}
$$

or

$$
G(j)=G^{f}(j)+\mathrm{O}\left(\frac{\partial}{\partial t}\right)
$$

These operations can be represented graphically with Feynman diagrams, in which $t$ is an interaction term and $s$ is like a mass term. This leads to a series of diagrams for $G$. These diagrams have no loops.

The quantities computed as described above depend upon the specific realization of the noise. Since we are interested in average quantities, we will compute correlation functions. A correlation function is defined as an average over noise of a product of several $G(j), G^{*}(j)$ with the same total number of $G$ and $G^{*}$, and will be written as

$$
\left\langle G\left(j_{1}\right) G\left(j_{2}\right) \ldots G\left(j_{n}\right) G^{*}\left(k_{1}\right) G^{*}\left(k_{2}\right) \ldots G^{*}\left(k_{n}\right)\right\rangle
$$

When computing these averages, the average over noise must be taken. This is done by taking noise terms resulting from the above expansion for $G$ and $G^{*}$ and contracting them with each 
other in all possible pairwise fashions. Each contraction of two noise terms at momenta $j, k$ leads to a factor of $\delta(j-k) N(j)$, as given by equation (33). This leads to diagrams with loops.

Fig. 3 indicates how diagrams for the theory are drawn. There are three types of diagrams that may be drawn. They are drawn in essentially the same fashion, except that different meanings are assigned to the external lines and different numbers of noise contractions are included. A diagram for a correlation function has one external line for each term in the correlation function to be computed. Diagrams for quantities other than correlation functions may have different meanings assigned to the external lines. These diagrams all involve one or more external lines being expressed as a function of the other external lines. This may occur either as an expansion of $G$ directly in terms of the noise, or as a piece of a diagram that occurs inside another diagram. For example, the diagram (a) in Fig. 3 is not itself a correlation function, but it represents a term that may occur inside a computation of a correlation function. For this diagram, the line for $G(j)$ is expressed in terms of other lines, which in turn may be set equal to the noise, or may be further expanded.

The notation for diagrams is the following: crosses denote contractions on noise, and the directions of the lines indicate complex conjugation and orientation within the diagram. A line may be said to carry momentum $j$; when two lines are contracted, they must carry the same momentum and all external lines are assigned a momentum determined by the particular correlation function to be computed. A $t$ vertex has 2 lines entering and 2 lines leaving, corresponding to $G$ and $G^{*}$. The $G(j)$ line for a $t(j, k)$ vertex is drawn as entering, the $G^{*}(m)$ is drawn as entering since it is complex conjugated, while the other lines are drawn as leaving the vertex. The $j$ and $k$ lines will always be drawn parallel to each other in a $t$ vertex; the $l$ and $m$ lines will be drawn at an angle. The distinction must be made between $j, k$ and $l, m$ because $j$ and $k$ enter into $t$ while $l$ and $m$ are summed over blindly.

The rules for diagrams for correlation functions may be summarized as follows: to calculate the average of the product of $G\left(j_{1}\right) G\left(j_{2}\right) \ldots G\left(j_{n}\right) G^{*}\left(k_{1}\right) G^{*}\left(k_{2}\right) \ldots G^{*}\left(k_{n}\right)$, where $j_{i}, k_{i}$ are various numbers: draw one external line for each term in the correlation function. The terms in $G$ should be drawn as entering the diagram with momentum $j_{i}$, while those for $G^{*}$ should be drawn as leaving the diagram with momentum $k_{i}$. Draw all possible diagrams, assigning a factor of $t(j, k) 2 \theta(j-k)$ to each vertex and a factor of $1 / s(j)+\mathrm{O}\left(\frac{\partial}{\partial t}\right)$ for each line, while conserving the sum of ingoing and outgoing momentum at each vertex. For each noise contraction assign 
a factor of $s^{2}(j) N(j)$. Finally, the perturbation theory may give rise to a term such as $\theta(0)$. This will arise from something like $\int d j \delta(j-k) \theta(j-k)$. While $\theta(j)$ is 0 for negative $j$ and 1 for positive $j, \theta(0)$ will be taken to be equal to $1 / 2$, according to equation (15). This is a result of the factor of two difference between powers of $z$ in the expansion for $f$; this difference was discussed in reference to equation (9). Alternately, if we recall that all $\delta$ functions should be assigned some nonzero width, then the above integral evaluates to $1 / 2$.

\subsection{Resummation}

This theory exhibits a spontaneous breaking of circular symmetry. One starts the growth process with a circularly symmetric cluster, which implies that $G(j)=0$ for $j>0$, but this is not a stable state. Instead, the dynamics evolves $G$ to one of an infinite number of states with nonzero $G(j)$, although on average $G(j)=0$ for $j>0$. If we impose some boundary condition, such as $G=1$ at time $t=0$, and look at $G$ for much later times, the perturbation theory in a small noise term causes us to to reach large values of $G$. Having imposed these boundary conditions, the noise is amplified by the dynamics and will grow large.

This large growth of noise means that if we evaluate the two-point correlation function $\left\langle G(j) G^{*}(k)\right\rangle$ we will obtain some answer of the form $\delta(j-k)\left|G^{2}(j)\right|_{a v}$, where $\left|G^{2}(j)\right|_{a v}$ is an appropriate function of $j$, and $\left|G^{2}(j)\right|_{a v}$ is much larger than $N(j)$. In the long time limit, $\left|G^{2}(j)\right|_{a v}$ is not a function of time.

Then, we may imagine that, when calculating any other correlation function, at any stage in the perturbation theory, two lines which were contracted to obtain the value $N(j)$, can instead have their contraction dressed with additional interactions to convert $N(j)$ to $\left|G^{2}(j)\right|_{a v}$. Two important points must be made about this procedure. First, it is important that this value $\left|G^{2}(j)\right|_{\text {av }}$ is completely uncorrelated with any other values of $G$ in the diagram; all correlations are already taken into account by the interaction vertices of the diagram. Second, we must be careful to avoid overcounting; since each contraction in a diagram includes many diagrams involving dressing the contraction in various ways, we must not further dress these contractions.

For notational convenience, I will continue to write $G^{f}$ everywhere, but now

$$
\left\langle G^{f}(j) G^{f *}(k)\right\rangle=\delta(j-k)\left|G^{2}(j)\right|_{a v}
$$

$G^{f}$ is similar to a free field in field theory. Any average product of several $G^{f}$ can be decomposed into pairwise products. This is the resummation of noise into two-point correlation functions of 
$G$ that was mentioned above in the comparison to Navier-Stokes perturbation theory. $G^{f}(j)$ is now an unknown function of $j$; one of the goals of the RG will be to determine the dependence of this function on $j$.

Similarly, the function $s(j)$ can be resummed. Any single line between two interaction vertices, which would normally be represented by a factor of $1 / s$, will instead be represented by $1 / s^{e f f}$, where $s^{\text {eff }}$ takes into account possibilities of dressing that line, without interactions with other lines. $s^{e f f}$ is an "effective" $s$. This is the resummation of propagators.

As a result of these two resummations, to avoid overcounting we must require that we do not count diagrams in which some portion of the diagram that contains interactions has only two lines leaving it. See Fig. 4 for examples of contributions to $j$, contributions to $s^{\text {eff }}$, and diagrams which can not be included in the theory as they would overcount contributions.

As one will be able to verify after performing the RG calculation, within the formal power series expansion of $\left(s+\frac{\partial}{\partial t}\right)^{-1}$, one may neglect all terms in $\frac{\partial}{\partial t}$ in the RG calculation of the next section. Terms only flow to higher powers of $\frac{\partial}{\partial t}$. Because $\left|G^{2}(j)\right|_{a v}$ is taken to be constant in time, terms with $\frac{\partial}{\partial t}$ will drop out in many places.

Then, the perturbation rules, ignoring time derivative, may be summarized as follows: draw all diagrams (subject to the rules forbidding overcounting), with appropriate external lines, assigning a factor of $1 / s^{e f f}(j)$ to each line, and a factor of $t(j, k) \theta(j-k)$ to each vertex, and a factor of $\left(s^{e f f}\right)^{2}(j)\left|G^{2}(j)\right|_{a v}$ to each contraction on noise, while conserving momentum everywhere.

\subsection{Example Calculation}

It will be useful to give a simple example of applying such a perturbation theory to a noninteracting system. For example, consider the following simplified equation of motion:

$$
G_{t}(j)+s G(j)=t(j, j) G(j) G(0) G^{*}(0)+s G^{f}(j)
$$

where $t(j, j)$ is proportional to $j-2 j-1$ which is equal to $-j-1$. Let us suppose $G^{f}$ is constant in time, to simplify the problem further. Then, let us define the time scale so that $t(j, j)=-j-1$. Let us pick the desired scale of the cluster so that $G(0)=1$. Physically, $G(0)$ is very large compared to the noise $G^{f}(0)$. This mean that for a stationary state (after all, $s$ is adjusted to produce a stationary average size) we may let $G^{f}(0)$ be small and we need $s$ approximately equal to -1 . 
Now that the values of $s, t$ are fixed, we may find the solution, either using perturbation theory or using a straightforward solution. The latter method gives $-G(j)=-G(j)-j G(j)-$ $G^{f}(j)$. Therefore, $G(j)=G^{f}(j) / j$ and $\left\langle G(j) G^{*}(j)\right\rangle=N(j) / j^{2}$. This noninteracting system is stable.

The perturbation theory for $G(0)$ is slightly tricky. If we ignore the time derivative, we can only reach small values of $G(0)$ in the perturbation expansion. However, in fact, for the given $s$ and $t$, a small $G^{f}(0)$ produces a large $G(0)$, under the time evolution. Let us suppose that this part of the process has been done, and that we obtain a resummed expression for $\left\langle G(0) G^{*}(0)\right\rangle$. This resummed expression is $\left|G^{2}(0)\right|_{a v}=1$. Then, we may ignore the time derivatives and obtain the expression for the higher $G(j)$ in a straightforward fashion.

The perturbation theory expansion to $G(j)$ gives the following infinite sum:

$$
G(j)=G^{f}(j)+\left(\left|G^{2}(0)\right|_{a v} t(j) / s\right) G^{f}(j)+\left(\left|G^{2}(0)\right|_{a v} t(j) / s\right)^{2} G^{f}(j)+\ldots
$$

This is equal to $G^{f}(j)\left(1-\left|G^{2}(0)\right|_{a v} t(j) / s\right)^{-1}=G^{f}(j) / j$. This perturbation expansion is shown in Fig. 5. Each line terminates by setting $G$ equal to the noise. Similarly, the perturbation expansion to the correlators gives a product of two infinite sums. This product is represented in diagrams by taking the sum in Fig. 5 and noise contracting it with its complex conjugate.

One may define $s^{e} f f(j)$ for this simple theory; the diagrams of Fig. 5 define the inverse of $s^{e f f}$. Therefore

$$
s^{e f f}(j)=s-t(j)\left|G^{2}(0)\right|_{a v}
$$

which simplifies equation (43) to $G(j)=\frac{1}{s^{e f f}(j)} s G^{f}(j)$.

\section{Renormalization Group Calculation}

We investigate the effect of changing the cutoff in the equation of motion. This leads to the introduction of new diagrams to describe the changes in the theory as a result of lowering cutoff. It is shown how to incorporate these into a change in $s, t$. The fixed point is found.

\subsection{Lowest Order Contributions to RG Flow}

In the RG calculation, first the cutoff is lowered from $\Lambda$ to $\Lambda-\delta \Lambda$. If the cutoff is imposed in a smooth fashion (interaction term $t(j, k) G(k) G(l) G^{*}(m) \exp [-(l+m) / \Lambda]$ ), the change in the 
theory under a change in the cutoff can be obtained by adding an additional term to the equation of motion (35), equivalent to the original cubic term, except that the cutoff $\exp [-(l+m) / \Lambda]$ is replaced by $(\delta \Lambda) \partial(\exp [-(l+m) / \Lambda]) / \partial \Lambda$. This new term is

$$
\begin{aligned}
& (1 / \mu) \int d k t(j, k) G(k) \iint d l d m G(l) G^{*}(m)(\delta \Lambda / \Lambda)(-(l+m) / \Lambda) \exp (-(l+m) / \Lambda) \\
& \times \delta(k+l-m-j) 2 \theta(j-k)
\end{aligned}
$$

The sum of the original interaction term plus this new term is equal to the interaction term at reduced $\Lambda$. This term will have a circle around the vertex when it appears in a diagram.

Because this term is small when the external momenta $l, m$ are small, it does not directly enter into correlation functions of the low momentum theory. It enters indirectly, in more complicated diagrams. We will then consider various such diagrams which include this term, and show that, for the simplest such diagrams, these diagrams may be rewritten in terms of a change in $s$ and $t$.

This procedure has some slight logical differences with other RG procedures. In other procedures, the cutoff is often imposed in a sharp fashion at some momentum. Here, the cutoff is imposed in a very smooth fashion; I believe this has certain logical advantages. This procedure is similar to the technique of counterterms in the original formulation of renormalization in field theory.

First, I will evaluate the simplest diagrams to which this new term gives rise, and do the RG calculation, then in the next subsection I will consider other possible diagrams and explain why they are neglected. Throughout the RG calculation, when I write $\left|G^{2}(\Lambda)\right|_{a v}$, I actually mean

$$
\int d l\left|G^{2}(l)\right|_{a v} \frac{\partial e^{-2 l / \Lambda}}{\partial \Lambda}
$$

This is just a weighted sum of $\left|G^{2}(l)\right|_{a v}$ at $l$ of order $\Lambda$, and due to the smooth cutoff it is this weighted sum that will enter into all the diagrams considered.

The diagrammatic expansion for $s^{\text {eff }}$ will now include the diagram shown in Fig. 6. This changes $s^{e f f}(j)$ to

$$
s^{e f f}(j)-t(j, j)\left|G^{2}(\Lambda)\right|_{a v} \delta \Lambda / \mu
$$

The term $2 \theta(j-k)$ in equation (45) gives 1 in this case, as discussed in the perturbation theory rules. 
Additionally, the new term in the equation of motion can give rise to a diagram as shown in Fig. 7a, which can be represented by changing $t(j, k)$. This arises from substituting

$$
s^{e f f}(l) G(l)=G^{f}(m) t(l, m) G(n) G^{*}(o) \delta(m+n-l-o) 2 \theta(l-m)
$$

and

$$
G^{*}(m)=G^{f *}(m)
$$

into equation (45), taking $l$ of the order of $\Lambda$. The result changes $t(j, k)$ to

$$
t(j, k)+2(\delta \Lambda / \mu) t(j, k) t(\Lambda, \Lambda+k-j)\left|G^{2}(\Lambda+k-j)\right|_{a v} / s^{e f f}
$$

Assuming $\Lambda$ is high momentum and $j, k$ are low momentum, then $\Lambda+k-j=m$ is high momentum. One does not use terms arising from substituting $s^{e f f}(l) G(l)=G(n) t(l, n) G^{f}(m) G^{*}(o)$, instead of the substitution of equation (48), because such terms involve too many high momentum components of $G$. The diagram corresponding to this term is shown in Fig. 7b. Such terms should be ignored, as they will be small when determining the behavior of the system for momenta much less than $\Lambda$. Remember that the cutoff is imposed, in the original equation, on $G(l)$ and $G^{*}(m)$, not $G(k)$. This means that the presence of the high momentum term $G^{*}(m)$ will make such terms small.

All momenta are now rescaled by $\frac{\Lambda}{\Lambda-\delta \Lambda}$ to put the ultraviolet cutoff back at $\Lambda$. This changes $s^{e f f}(j)$ to

$$
s^{e f f}(j)-j\left(d s^{e f f}(j) / d j\right)(\delta \Lambda / \Lambda)
$$

Because of the integration in the interaction term, and the one power of $\mu$ extracted from $t(j, k)$, the dimension of $t(j, k)$ is equal to (momentum $)^{-1}$. Therefore, under rescaling, the function $t(j, k)$ becomes

$$
t(j, k)-j(\partial t(j, k) / \partial j)(\delta \Lambda / \Lambda)-k(\partial t(j, k) / \partial k)(\delta \Lambda / \Lambda)-t(j, k)(\delta \Lambda / \Lambda)
$$

Combining the terms resulting from the integration, equation (50), with those from the momentum rescaling, equation (52), every term in the change of $t(j, k)$ either is a function of $(j-k)$, or would be a function of $(j-k)$ if $t(j, k)$ were a function of $(j-k)$. For a stationary point, then require that

$$
t(j, k)=t(j-k)
$$


If $t(j, k)=t(j-k)$, then similar logic using equations (47), (51) requires that $s^{\text {eff }}(j)$ becomes a constant. These requirements of momentum independence of $s, t$ likely do not hold at the extreme infrared for any true system, or else the equation of motion (equation (35) using renormalized $t$ and $s$ ) would have no non-zero solution, but in the scaling region between the infrared and ultraviolet, they will hold.

For the rest of this subsection, the number $s$ is the constant to which $s^{\text {eff }}$ flows under the $\mathrm{RG}$, and the number $t$ is $t(0)$.

At this point, I have done the first part of the RG for the two numbers $s$ and $t$. It is now necessary to rescale $G$ to leave equation (35) invariant under the renormalization and rescaling. Let $G$ be rescaled to

$$
(1-r(\delta \Lambda / \Lambda)) G
$$

This implies a rescaling of $s$ to

$$
(1+r(\delta \Lambda / \Lambda)) s
$$

and a rescaling of $t$ to

$$
(1+3 r(\delta \Lambda / \Lambda)) t
$$

Naively one might expect that there could also be an overall rescaling of both $s$ and $t$ by a factor $a$. This would leave $G$ unchanged. Taking derivatives of $\log (s)$ and $\log (t)$ with respect to $\log (\mu)$ (since the ultraviolet cutoff is lowered to $\Lambda-\delta \Lambda$ and then rescaled back to $\Lambda$, it is actually $\mu$ that changes in this process), for $s$ and $t$ to be stationary we find:

$$
\begin{gathered}
r-(\Lambda / \mu) t\left|G^{2}(\Lambda)\right|_{a v} / s+a=0 \\
3 r+2(\Lambda / \mu) t\left|G^{2}(\Lambda)\right|_{a v} / s+a-1=0
\end{gathered}
$$

This in itself does not provide enough information to extract anything useful, because having both $r$ and $a$ means that $s$ and $t$ can be scaled to arbitrary values.

However, in fact, $a=0$, as will now be shown. The time derivatives of equation (35) were ignored in the RG because they did not effect the flow of $s$ and $t$. We can still use arguments about the time derivative, $G_{t}(j)$, to show that $a=0$. We can use equation (35) to calculate $G_{t}(j)$ of the renormalized, rescaled problem in two ways. One way is to to take $G_{t}(j)$ for the problem before renormalization and rescaling, and then rescale the momentum $j$ and multiply 
$G_{t}(j)$ by $(1-r(\delta \Lambda / \Lambda))$ in analogy with equation (54). Another way is to use the renormalized, rescaled $s, t$, and $G^{f}$ in equation (35) to compute $G_{t}(j)$. For these two methods of computing $G_{t}(j)$ to give the same result, as required by the fact that they describe the same system, it may be shown that

$$
a=0
$$

A simple way of stating the argument leading to this result is that, although if time derivatives are neglected in equation (35) the equation is invariant under multiplying the three terms $s, t$, and $G^{f}$ (here referring to the noise before resummation, that is, the noise described by equation 33) by the same constant, such a multiplication does not leave the time derivatives invariant and so should not be allowed in the rescaling part of the RG.

Then, the fixed point equations (57),(58) can be solved for

$$
r=(\Lambda / \mu) t\left|G^{2}(\Lambda)\right|_{a v} / s=1 / 5
$$

This result for $r$ is the main result of the first order RG.

This gives the rescaling of $G(j)$ with $j$. The magnitude of $G(j)$ must decay as $j^{-1 / 5}$. Thus

$$
\left|G^{2}(j)\right|_{a v} \propto j^{-2 / 5}
$$

The combination $(\Lambda / \mu) t\left|G^{2}(\Lambda)\right|_{a v} / s$ is invariant under a rescaling of $G$ of $G$ by equation (54) and a corresponding rescaling of $s$ and $t$ by equations (55),(56), and gives us the dimensionless coupling constant for this problem. From equation (60) the dimensionless coupling constant is $1 / 5$, which is not infinitesimal; however, after $\mathrm{RG}$ flow, the problem is no longer strongly coupled, as the constant is less than 1 .

The reason that a factor of $1 / \mu$ was removed from $t(j, k)$ is now clear; this makes the above coupling constant truly dimensionless. As a result of the removal of the factor of $1 / \mu$, the dimension of $t(j, k)$ is (momentum) $)^{-1}$. However, $\left|G^{2}(j)\right|_{\text {av }}$ has the dimension of momentum; this is because, taking $G$ to be dimensionless, equation (41) gives $\left|G^{2}(j)\right|_{a v}$ a dimension inversely proportional to the $\delta$-function. The $\delta$-function has dimension of inverse momentum, and thus the end result is to make $t\left|G^{2}(j)\right|_{\text {av }}$ dimensionless.

As a further comment on the dimensionality of the coupling constant, recall that the $\delta$-functions have a finite height proportional to $1 / \mu$. This finite height changes under the RG, 
which implies a rescaling of $\left|G^{2}(j)\right|_{\text {av }}$ under the RG flow; by multiplying $t$ by $1 / \mu$ we shift this rescaling onto $t$.

An argument was made above, leading to equation (59), involving $G_{t}$ for the problem before and after RG. We may extend this argument and also say something about the magnitude of $G_{t}(j)$ for different $j$. The quantitities $s G$ and $t G^{3}$ remained constant under the RG, as a result of the rescalings of $G, s$, and $t$ and the renormalizations of $s$ and $t$, from equations (47),(50),(51),(52),(54). We also need $G_{t}$ to remain constant since this is also a term in equation (35). Suppose the characteristic inverse time scale for fluctuations in $G(j)$ is $\omega(j)$. Then $G_{t}(j)$ is of order $\omega(j) G(j)$. For this combination to remain constant, $\omega$ must change as a result of the rescaling of $j$ in the RG. In fact, $\omega(j)$ must have the same log derivative under RG that $s$ does, although the log derivative of $s$ results from renormalization (equation 47) while the log derivative of $\omega(j)$ results from rescaling. This implies that

$$
\omega(j) \propto j^{1 / 5}
$$

This means that for smaller $j$, the time scale for fluctuations is longer. Returning to the original problem, as described by equation (29) with a time-dependent cutoff, this means that the lower Fourier coefficients grow at a roughly constant rate. This self-consistently justifies the adiabatic assumption of section 3 .

\subsection{Other Contributions to RG Flow}

One may imagine that the new term of equation (45), representing the rescaling of the cutoff, may enter into additional diagrammatic contributions. Various possibilities are shown in Fig. 8. I will show that, for low momentum behavior, these terms are unimportant and then discuss in more generality why other contributions are negligible.

One may check by hand that the first example is small if external momenta are much less than $\Lambda$. The second example will be discussed below. The third example vanishes due to phase space factors. The fourth example vanishes due to phase space factors if the two lines leaving the top of the diagram are close in momentum; this means it does not alter the RG flow of $t(j, k)$ when $j=k$. In the rest of the section, various other diagrams will also be said to "vanish"; this will only mean that they vanish when considered either at low momentum or, if they contribute to the RG flow of $t(j, k)$, when considered at $j=k$. 
The fifth example, a contribution to the six-point function, will be seen below to be small when calculating correlations of only 4 , and not 6 or more, $G(j)$. The sixth example should not be considered when the rescaling of $\left|G^{2}(j)\right|_{a v}$ is taken into account; since $(\Lambda / \mu)\left|G^{2}(\Lambda)\right|_{a v}$ is stationary under RG flow, such a diagram is canceled by the various rescalings.

In order to indicate in general why such contributions may be neglected, I would like to define some additional terminology to describe certain paths and contractions in these diagrams. When considering a contribution to $s^{e f f}$, one may follow one line through the diagram as follows: start with the incoming line. At every $t$ vertex, if one enters with $G(j)$, follow out along $G(k)$, not $G(l)$ or $G^{*}(m)$, where the roles of $j, k, l, m$ are as in equation (35). If the line one is following is contracted (this will be referred to as an exceptional contraction) with a $G^{*}(m)$ leaving a $t$ vertex, follow out along $G(l)$ of that $t$ vertex. This path will be referred to as the main line. Now, any diagram that includes an exceptional contraction, such as the diagram of Fig. 8a, will be small for low momenta, since the $G(k)$ leaving such a diagram will only have a small range over which to integrate. For Fig. 8a the main line is given by following the horizontal arrow along the bottom of the diagram from left to right, through the $t$ vertex, until it bends up and left. Then go down and left through the noise contraction into the $t$ vertex, and then leave the $t$ vertex along the line going up and left. Follow this line through its bend back to the right until it leaves the diagram.

The smallness, of the contribution to $s^{e f f}$ given in Fig. 8a, for small momenta does not completely justify the neglect of such terms. For example, when evaluating the RG contribution to $t$, the value of $s^{e f f}$ used is $s^{e f f}(\Lambda)$, not the low momentum $s^{e f f}$, and thus a high momentum contribution to $s^{\text {eff }}$ may change the low momentum renormalized $t$. However, even for a calculation of $s^{e f f}(\Lambda)$, the exceptional contraction will mean some reduction in available phase space over which to integrate.

One may check that contributions to $s^{e f f}(j)$ like the third example in Fig. 8 will always vanish, regardless of what $j$ is, due to the $\theta$ functions in equations (35),(45). The lines coming off of $t$ vertices connected to the main line must be contracted within themselves, not between different vertices. In this diagram, the main line is simply the entire horizontal arrow running along the bottom of the diagram. Thus, the only contribution to $s^{\text {eff }}$ will be the contribution of Fig. 6.

For contributions to $t(0)$, we may define 2 main lines. One can follow the main line of 
the $G(l)$ or the main line of the $G^{*}(m)$. These are the lines one follows if one starts on the line for $G(l)$ or for $G^{*}(m)$ and follows through the various contractions as defined above. These two lines join at some point in a noise contraction.

The second example of Fig. 8 has an exceptional contraction and may be ignored. The main line starting with the $G(l)$ line leaving the circled $t$ vertex proceeds up and left, then turns right, going straight across the diagram to the end. Then it turn down and left, up and left through an exceptional contraction into a $t$ vertex. Then it goes down and left into a noise contraction, where it ends. The main line starting with $G^{*}(m)$ starts at the circled $t$ vertex and proceeds up and right until it terminates at the noise contraction. Fig. $8 \mathrm{~b}$ is very like Fig. $7 \mathrm{~b}$, except an additional $t$ vertex has been added to the diagram. If this vertex were removed, this diagram would be small for small external momenta. With the $t$ vertex on the diagram, the diagram is very difficult to evaluate since to evaluate it requires a knowledge of all $t(j, k)$, not just $t(\Lambda, \Lambda)$. However, the diagram is not only next order in the coupling constant, but also small due to the various exponentials present, as may be verified. Any diagram for $t$ with such an exceptional contraction will have the same problems. That is why we will ignore them.

If the main line of the $G^{*}(m)$ has lines leaving it which contract against lines leaving the main line of $G(l)$ then the diagram will again vanish due to the $\theta$ functions. A contribution to $t$ cannot have both external lines leaving the main line of $G^{*}(m)$. Therefore both lines must leave the main line of $G(l)$, and the line $G^{*}(m)$ cannot be dressed by any interaction vertices. Again due to $\theta$-functions, the main line of $G(l)$ can only include one interaction vertex which has both external lines on it. For example, the diagram of Fig. 8d has external lines coming off different interaction vertices and vanishes if the two lines are close in momentum.

All that remains is to justify the neglect of six-, and higher-,point functions, such as could appear from the diagram in Fig. 8e. If we wish to calculate a correlation function of four $G(j)$, and somewhere in one diagrammatic calculation we have a six-point function, some of the lines leaving the six-point function must be contracted against each other. Then, somewhere in the diagram for the correlation is a contribution to the four-point function which includes the six-point function within it. Therefore, the renormalization procedure would have yielded this contribution to the four-point function as a change in $t$. But, we have already, as outlined above, obtained all the contributions to the change in $t$. Therefore, there is no such diagram.

Finally, the lowest order contributions considered in the previous subsection have a 
certain universality; considering only the diagrams of that subsection, the nature of the fixed point does not depend on the initial form of $t(j, k)$. Higher order RG contributions will depend on the initial form of $t(j, k)$.

\section{Fractal Dimension and Multifractal Exponents}

It is now possible to begin extracting exponents of the original DLA model. Different exponents correspond to different correlation functions of this model; it will be the purpose of this section to determine how to compute exponents from correlation functions. This process depends on the discussion of the adiabatic assumption and the assumption used to introduce the cutoff into the continuum equation. From those assumptions an unambiguous means of determining exponents from correlation functions is given.

In any actual simulation, there is an ultraviolet cutoff $\Lambda$ determined by the ratio of macroscale to microscale. In the RG, a power law decay was found for $G(j)$. Since the RG describes a fixed point in the scaling region, within the RG itself this power law holds for arbitrarily large $j$. Within an actual simulation this power law will fail at $j$ of order $\Lambda$, where $\Lambda$ is the cutoff of equation (25) resulting from the finite size of the cluster in the simulation. Thus, in the calculation of exponents that follows, although all correlation functions are calculated using the rules of the RG and of perturbation theory, the integrals over correlations functions that we will use must be cutoff at momenta of order $\Lambda$, as will be done.

Since the size of the object follows a power law behavior given by

$$
F_{1} \propto t^{1 / D}
$$

where $D$ is the radius of gyration dimensions of the object, we have

$$
\frac{d \log \left(F_{1}\right)}{d t} \propto 1 / t
$$

As a side point, strictly speaking this requires that $d F_{1} / d t$ can be replaced by the derivative of the average value of $F_{1}$, but both numerical evidence and the RG flow of frequency resulting from equation (62) justify this assumption. However,

$$
d F_{1} / d t=\langle\lambda\rangle F_{1}
$$


where $\langle\lambda\rangle$ is defined to be the average value of $\lambda$ over the unit circle at a given time. Equation (65) may be derived by using equation (14) to calculate $d G_{0} / d t$ and then equation (26) to relate this to $d F_{1} / d t$. Combining equations (64),(65) we get

$$
\langle\lambda\rangle \propto 1 / t
$$

Equation (66) is equivalent to the electrostatic scaling law first derived by Halsey [5]. In the continuum approximation, $\langle\lambda\rangle=\int d j\left\langle G(j) G^{*}(j)\right\rangle$. Here it must be understood that while in the perturbation theory this expression is formally infinite, since $\left\langle G(j) G^{*}(j)\right\rangle=\delta(0)\left|G^{2}(j)\right|_{a v}$, in the above average we remove this factor of $\delta(0)[17$. Calling $D$ the fractal dimension,

$$
F_{1}^{-D} \propto 1 / t \propto\langle\lambda\rangle \propto G_{0}^{2} \int^{\Lambda} d j j^{-2 / 5} \propto F_{1}^{-2} \Lambda^{3 / 5} \propto F_{1}^{-2} F_{1}^{3 / 10}
$$

This gives the result that

$$
D=2-1 / 2+1 / 5=1.7
$$

The first proportionality in equation (67) followed from the radius of gyration definition of the dimension. The second followed from the electrostatic scaling law. The third followed from the expression for $\langle\lambda\rangle$ in terms of $G$ and from the scaling of $G$ derived in the RG. The fourth followed from equation (26) and from doing the integral. The fifth followed from the functional dependence of $\Lambda$ on $F_{1}$ as given by equation (25).

This is the simplest way to derive the fractal dimension from the above work. The calculation of the growth rate from equation (66) is essentially a determination of the unrenormalized, unrescaled $s$ in equation (35). It may also be possible to repeat the same result by using the rescaling of $s$ under the RG to obtain the rescaling of the growth rate under a shift in $\Lambda$.

The multifractal exponents $\tau(q)$ are defined by

$$
\tau(q)=\lim _{l \rightarrow 0}\left(\log \left(\sum_{i} E^{q}(i)\right) / \log (l)\right)
$$

where the surface of the cluster is covered with intervals of length $l$, and $E^{q}(i)$ is the integral along the $i$ th interval of the $q$ th power of the electric field. Numerical calculations of these exponents can be found in references [10, 18].

One can try to compute higher multifractal exponents using the RG (the work above amounts to computing $\tau(3)$ and showing that $D=\tau(3)$ ). For example, the scaling of $\tau(5)$ can 
be determined by calculating the scaling of

$$
\int^{\Lambda} d j d k d l d m\left\langle G(j) G(k) G^{*}(l) G^{*}(m)\right\rangle \delta(j+k-l-m)
$$

against the upper cutoff $\Lambda$. This is because the given integral is equal to the desired power of the field integrated over the surface of the object. If all the terms in this integral contributed with the same phase, the integral would scale as $\Lambda^{-4 / 5+3}$. Of course, the terms are independent and this misestimates the exponent. It is necessary to use the perturbation theory to evaluate the 4 point correlation function. The simplest possibility is to use $G^{f}$ as an estimate for all the $G$ in equation (70). The diagram for this is shown in Fig. 9a. The only terms that would then contribute would be when a $G$ and a $G^{*}$ were at the same momentum and the integral would scale as $\Lambda^{-4 / 5+2}$. The different scaling results from having a different number of momenta to integrate over. Another possibility (this is analogous to a tree approximation for a scattering problem) is to substitute for the highest momentum $G$ in terms of a $t$ vertex, leaving a six-point correlation function, and then take all six $G, G^{*}$ to be $G^{f}$. The scaling is then as $\Lambda^{-6 / 5+3}$. The diagram for this is shown in Fig. 9b. Since this scales more strongly with $\Lambda$, it will be dominant in the limit needed to compute $\tau(q)$.

The following is the rule for calculating multifractal exponents: Let $n$ be a positive integer. Calculate the integral over 2n-point correlation functions defined by

$$
\begin{aligned}
& \int^{\Lambda} d j_{1} \int^{\Lambda} d j_{2} \ldots \int^{\Lambda} d j_{n} \int^{\Lambda} d k_{1} \int^{\Lambda} d k_{2} \ldots \int^{\Lambda} d k_{n} \\
& \left\langle G\left(j_{1}\right) G\left(j_{2}\right) \ldots G\left(j_{n}\right) G^{*}\left(k_{1}\right) G^{*}\left(k_{2}\right) \ldots G^{*}\left(k_{n}\right)\right\rangle \delta\left(j_{1}+j_{2}+\ldots+j_{n}-k_{1}-k_{2}-\ldots-k_{n}\right)
\end{aligned}
$$

If this integral behaves, in the limit of $\Lambda$ taken to infinity, as $\Lambda^{a}$, where a is some number, then $\tau(2 n+1)=(2 n)-a / 2$. The factor of $(2 n)$ is the trivial scaling that would result even for a nonfractal object; the factor of $a / 2$ results from the dependence on $\Lambda$ and from the square root dependence of $\Lambda$ on length scale.

In general, we can always find, for $\tau(q)$, a tree diagram that scales like $\Lambda^{-2(q-2) / 5+(q-2)}$. Then,

$$
\tau(q)=(q-1)-1 / 2(-2(q-2) / 5+(q-2))
$$

Alternately, another definition of exponents is

$$
D_{q}=\tau(q) /(q-1)
$$


Then

$$
D_{q}=\tau(q) /(q-1)=1-\frac{q(1 / 2-1 / 5)-1+2 / 5}{(q-1)}
$$

which is equivalent to

$$
D_{q}=\frac{0.7 q-0.4}{q-1}
$$

\section{Comparison With Numerics and Discussion}

The theory is compared with numerics, and further tests of the theory are proposed.

\subsection{Comparison With Numerics}

In previous work we found that the alternate formulation of DLA using analytic functions [6] produces clusters with appearance and dimension similar to those of clusters grown using the lattice formulation of DLA. As far as we can tell, the two formulations are equivalent when $\alpha=2$.

The simplest comparison with numerics is the dimension itself. 1.7 is very close to the accepted value of 1.71 .

Equation (75) for higher multifractal exponents is the same as the formula obtained with

a wedge model by Halsey et. al. [10], except that the wedge model left the quantities 0.7 and 0.4 as unknown constants to be fitted to numerics. They define quantities $f$ and $\alpha$, the dimension of the set on which the wedges exist, and the strength of the singularity (hopefully, the reuse of the symbol $\alpha$ will cause no confusion), and show that $D_{q}=\frac{\alpha q-f}{q-1}$. A numerical fit gave $\alpha=0.705$, $f=0.42$, while a comparison with equation (75) gives $\alpha=0.7, f=0.4$. It is now known that such a simple scaling law is not valid for large $q$ [18, and in the original paper of Halsey et. al. it was suspected that such a law would not hold.

The possible difference between theory and experiment here for large $q$ should not be construed as a flaw in the presented work. First, the above calculation is only a lowest order calculation. To higher orders, we may find a form for $t(j, k)$ which has nontrivial behavior. This may alter the results from the tree approximation to the correlation function used to compute the exponents. Second, we may find interesting behavior if we consider other contributions to the correlation exponents, beyond the tree diagrams used above. Third, although the neglect of the appearance of 6-point function was valid when considering the renormalization flow of 
$s$ and $t$, as discussed in reference to Fig. 8e,, such a neglect is not valid if one actually wishes to compute 6- and higher point correlation functions. Fortunately, such multi-point interaction terms are captive variables, in the sense that if one knows the behavior of $s, t$ under RG flow one may systematically determine the higher interaction terms that will appear. Fourth, the above derivation of multifractal exponents involved expressing the exponent in terms of correlation functions; this is only possible for odd multifractal exponents. Thus, in fact it is not possible to say anything about even exponents in any simple fashion.

Additionally, there exist some difficulties in numerical calculation of higher multifractal exponents. According to the branched growth theory of DLA [16], the time required to compute higher exponents is superexponential in the order of the desired exponent. Thus, the exact values of the larger exponents may not be precisely given by the numerical experiments. This mathematical difficulty may be the source of the controversy which appears to exist between the different numerical calculations of these exponents. For example, the value quoted for $\tau(3)$ by Ball et. al. is less than 1.6, which is definitely at odds with the electrostatic scaling law (believed to be exact from various numerical calculations), and with other numerical calculations of this exponent. The electrostatic scaling law says that $\tau(3)=D=1.71$. There also exists controversy about the precise value of the dimension of DLA, as mentioned in Ref. [2]. Thus, in fact, it is not clear exactly how large is the discrepancy between the above results and the numerical results.

It is also of interest to numerically check the scaling of $G(j)$. This was check for two cases. First, for a single cluster as described in the next paragraph; second, for an ensemble of clusters as described in the paragraph after that.

When $\int d j G(j) G^{*}(j) e^{-j / \Lambda}$ is plotted against $\Lambda^{0.6}$, where in reality the integral is a discrete sum, one expects to find a straight line behavior. This is what is found, as shown in Fig. 10, except that for large $\Lambda$ the curve flattens out, since the numerical calculation only included a finite number of terms. Also for small $\Lambda$, the curve flattens out at $F_{1}^{-2}$, which, in the long time limit, is vanishingly small compared to the full integral. For the finite cluster size of our simulation, $F_{1}^{-2}$ is not negligible. The clusters here were grown using the conformal mapping technique outlined previously. The coefficients of $G$ were computed with a numerical Fourier transform, by mapping a large number of points on the surface of the circle (in fact, slightly outside the circle, to improve numerical behavior) to the surface of the aggregate (again, 
to slightly outside the surface of the aggregate) and analytically calculating the derivative of the mapping for each point. This technique is not very efficient for growing large clusters, at least as presently implemented. It requires $\mathrm{O}\left(N^{2}\right)$ time to compute $N$ growth steps, but it is very easy to calculate coefficients of $G$ using this program. I only used aggregates of around 7000-10000 walkers.

As another check, 50 clusters of 6000 steps were simulated, and for each cluster, the coefficients of $G$ were computed. The squares of these coefficients were scaled by $G(0)$, the overall inverse cluster size, and then averaged together. In Fig. 11 the mean squares of $G(j)$ are plotted against $j$ on a log-log plot. Numerical difficulties made it impossible to accurately extract the slope in the scaling region. This scaling region extends from $j=5$ to $j=35$, or from $\log (j)=1.6$ to $\log (j)=3.5$. Theory predicts that this slope is $2 / 5=0.4$. The numerical slope is between 0.3 and 0.5 , using a least squares fit. The theory line is drawn onto the graph. As an additional check, another ensemble of clusters was simulated, with a different $\lambda_{0}$ and a different number of steps. Within the scaling region of that simulation, the slope of the mean squares behaved in the same fashion, and, additionally, the mean-square of $G(j)$, after scaling by cluster size, for given $j$, was the statistically the same for the two simulations.

\subsection{Discussion}

A theory has been presented based on the conformal nature of various Laplacian growth processes. A series of approximations were made that produced a modified continuum equation of motion; it is hoped that such an equation describes DLA, but even if it does not, it does describe some form of nontrivial Laplacian growth. A perturbation theory was developed for this equation, and resummed. To determine various terms in the perturbation theory, it was then necessary to use a renormalization group calculation. This has only been carried out to lowest order. It is a peculiar feature of this method that next order calculations are vastly more difficult than lowest order calculations, thus as yet there is no analytic calculation of higher order effects. Finally, the assumptions leading to the modified model were reversed, leading to calculations of quantities for DLA.

It would be worthwhile to look more closely at higher order corrections, if not analytically, at least qualitatively, to see what may happen. To lowest order, $t(j-k)$ flows to an everywhere positive function. Using the lowest order $t$ to compute the effect of higher order corrections will 
tend to lower the value for the dimension predicted by this theory. However, it is possible that in a more careful next order calculation, the interaction $t(j-k)$ flows to a function which is negative for large $(j-k)$, possibly increasing the predicted dimension. As mentioned above, next order effects depend in some way upon the initial functional form of $t(j, k)$. Lowest order effects do not.

Unfortunately, it is not possible to carry out a stability analysis of the fixed point of the lowest order RG. All that may be said from the above calculation is that if a fixed point exists, other than a trivial fixed point for which $t$ goes to zero, then this fixed point is described by this RG.

It would also be worthwhile to try to extend this technique to other Laplacian growth models, such as the dielectric breakdown model. For the dielectric breakdown model[11], different values of $\eta$ correspond, in the continuum limit, to different values of $\alpha$ in the conformal mapping model of section 2.1. The difference between the dielectric breakdown model and our model is that, away from the DLA case, our model uses the same growth probability over the surface and varying walker size, while the dielectric breakdown uses a varying growth probability and constant walker size. Although this alters the scale of the cluster in our case, we would expect the fractal dimension of the cluster grown at a given $\alpha$ with the conformal model to be the same as the dimension obtained from the dielectric breakdown model with $\eta$ equal to $\alpha-1$.

One might naively try to apply the technique above to the case of $\alpha$ different from 2 , by replacing equation (1) with the definition $G=F_{z}^{-\alpha / 2}$, and using an equation of motion similar to (35), with different initial $t(j, k)$. This would lead to physically absurd results, and is in fact different from defining $G=F_{z}^{-1}$ and using a modified equation of motion as described in the next paragraph. The difference is in how the noise term is inserted. It is important for the perturbation theory that products of $G^{f}$ may be pairwise decomposed, and this property means different things depending on whether $G=F_{z}^{-1}$ or $G=F_{z}^{-\alpha / 2}$. In the stochastic problem, each growth step produces a simple pole of $F_{z}^{-1}$ inside the unit circle; the angular coordinate of the pole is random, the radial coordinate is determined by $\lambda$. In the continuum limit of section 2.5 , the angular coordinate becomes the real value of $\theta$, while the radial coordinate becomes the imaginary value of $\theta$; the interior of the unit circle is replaced by the lower half plane. One may show, using Cauchy's theorem, that randomly inserting simple poles produces a pairwise decomposition property for the random noise in $F_{z}^{-1}$. Therefore, the equation (11) is the best 
definition of $G$.

One can handle the case of $\alpha$ different from 2 by using a modified equation of motion, although this may be difficult if $\alpha$ is not even. One would modify equation (35) by including higher powers of $G$ in the interaction term. For example, for $\alpha=4$, the interaction term would be of the form

$$
\begin{aligned}
& (1 / \mu) \int d k t(j, k) G(k) \iint d l d m d n d o G(l) G^{*}(m) G(n) G^{*}(o) \\
& \exp (-(l+m+n+o) / \Lambda) \delta(k+l+n-m-j-o) 2 \theta(j-k)
\end{aligned}
$$

This would probably be the most worthwhile test of the calculations of this paper; although the calculation for $\alpha=4$ is far more difficult than that for $\alpha=2$, it may still be tractable to lowest order. It would not be appropriate to attempt such a calculation in this paper. A few

preliminary calculations show that one obtains at least the physically correct result that the dimension of the $\alpha=4$ model is less than that of the $\alpha=2$ model; as yet the exact value at $\alpha=4$ is not calculated[19].

\section{Acknowledgements}

I would like to thank Leonid Levitov for working with me on our previous paper on the conformal mapping model, and for many useful discussions that helped clarify the ideas outlined above.

\section{References}

[1] T. A. Witten, Jr. and L.M. Sander, Phys. Rev. Lett. 47, 1400 (1981).

[2] This result is the accepted result from many radius of gyration calculations. A lower result for the surface dimension is claimed by F. Argoul, A. Arneodo, G. Grasseau, and H. Swinney, Phys. Rev. Lett. 61, 2558 (1988). Also see G. Li, L. M. Sander, P. Meaking, Phys. Rev. Lett 63, $1322(\mathrm{C})(1989)$.

[3] Tokuyama, M. and Kawasaki, K., Phys. Lett. 100A, 337 (1984); Honda, K., Toyoki, H., and Matsushita, M., J. Phys. Soc. Jpn. 55, 707 (1986).

[4] T. C. Halsey, Phys. Rev. Lett 72, 1228 (1994).

[5] T. C. Halsey, Phys. Rev. Lett. 59, 2067 (1987); Phys. Rev. A 38, 4789 (1988). 
[6] M. B. Hastings and L. S. Levitov, to be published.

[7] M.B. Mineev-Weinstein and R. Mainieri, Phys. Rev. Lett. 72, 880(1994).

[8] B. Shraiman and D. Bensimon, Phys. Rev. A30, 2840 (1984).

[9] Z. Nehari, Conformal Mapping, Chap.V, Sect.4, (Dover, N.Y., 1975).

[10] T. C. Halsey, P. Meakin, and I. Procaccia, Phys. Rev. Lett. 56, 854 (1986).

[11] L. Niemeyer, L. Pietronero, and H.J. Wiesmann, Phys. Rev. Lett. 52, 1033 (1984).

[12] H. G. E. Hentschel and I. Procaccia, Physica (Amsterdam) 8D, 835 (1983)

[13] I. Procaccia, Phys. Scr. T9, 40 (1985)

[14] P. Meakin, H. E. Stanley, A. Coniglio, and T. A. Witten, Phys. Rev. A 32, 2364 (1985).

[15] H. W. Wyld Jr., Ann. Phys. 14, 143 (1961); D. Forster, D. R. Nelson, M. J. Stephen, Phys. Rev. A 16, 732 (1977).

[16] T. C. Halsey, K. Honda, B. Duplantier, to be published.

[17] Actually, $\delta(0)$ is of order $1 / \mu$, and so this formal infinity is quite correct. When integrating the electric field over the circle, the result should be proportional to the size of the circle. From the point of view of the continuum limit of section 2.5, this size is infinite, but in reality it is cut off at size $1 / \mu$.

[18] Various authors have calculated these exponents. For example, R. C. Ball, O. Rath Spivak, J. Phys. A 23, 5295 (1990) and references therein; C. Armitrano, Phys. Rev. A 39, 6618 (1989).

[19] For $\alpha \neq 2$, equation (20) gets replaced by $\operatorname{Re}\left(F_{t} \frac{\left|F_{z}\right|}{z F_{z}}\right)=\left|F_{z}\right|^{1-\alpha}-\alpha\left|F_{z}\right|^{2-\alpha} \epsilon \operatorname{Re}\left(\frac{z F_{z z}}{F_{z}\left|F_{z}\right|}\right)$ and so the balancing of electric field and surface tension of equation (23) is at the same value of $\epsilon$. It is expected then, that equation (25) is that appropriate equation for any value of $\alpha$. We found from equation (20) that $\delta=\epsilon$. Since $\delta$ is the distance of the zero of $F_{z}$ to the boundary of the unit circle, and $R \propto 1 / \delta^{2}$, this suggests that equation (25) may have a geometrical explanation, as well as a dynamical explanation. 